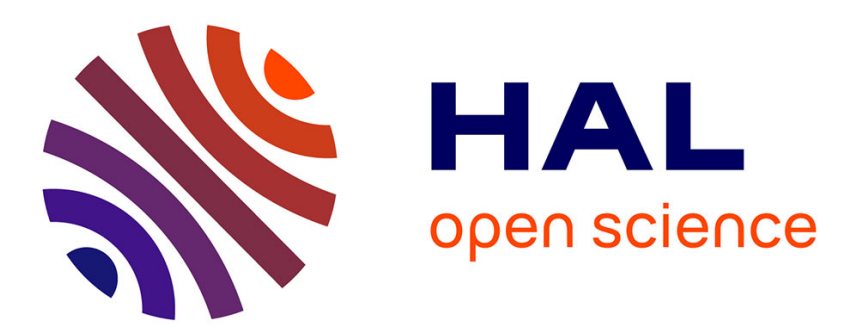

\title{
Estimation of Elastoplastic Parameters via Weighted FEMU and Integrated-DIC
}

Florent Mathieu, Hugo Leclerc, François Hild, Stéphane Roux

\section{To cite this version:}

Florent Mathieu, Hugo Leclerc, François Hild, Stéphane Roux. Estimation of Elastoplastic Parameters via Weighted FEMU and Integrated-DIC. Experimental Mechanics, 2015, 55 (1), pp.105-119. 10.1007/s11340-014-9888-9 . hal-01166755

\section{HAL Id: hal-01166755 \\ https://hal.science/hal-01166755}

Submitted on 23 Jun 2015

HAL is a multi-disciplinary open access archive for the deposit and dissemination of scientific research documents, whether they are published or not. The documents may come from teaching and research institutions in France or abroad, or from public or private research centers.
L'archive ouverte pluridisciplinaire HAL, est destinée au dépôt et à la diffusion de documents scientifiques de niveau recherche, publiés ou non, émanant des établissements d'enseignement et de recherche français ou étrangers, des laboratoires publics ou privés. 


\title{
Estimation of Elastoplastic Parameters via Weighted FEMU and Integrated-DIC
}

\author{
Florent Mathieu • Hugo Leclerc . \\ François Hild • Stéphane Roux
}

Received: date / Accepted: date

\begin{abstract}
DIC-based identification of the constitutive parameters of an elastoplastic law is addressed both from a general viewpoint, and applied to the particular case of dog-bone sample made of commercially pure titanium and subjected to tensile loading. A two-step procedure (Digital Image Correlation - DIC - followed by weighted Finite Element Method Updating FEMU) is first presented. These two steps can be merged into a single-step procedure (i.e., Integrated-DIC or I-DIC). In both cases, the elastoplastic computations are performed with a commercial code (i.e., non-intrusive identification). When the suited weighting of FEMU is taken into account, which is based on DIC-processed image noise, both I-DIC and FEMU methods provide similar results. It is shown that the addressed experimental case requires the use of static (load) information to get precise estimates of the sought parameters.
\end{abstract}

Laboratoire de Mécanique et Technologie (LMT-Cachan)

ENS Cachan / CNRS / PRES UniverSud Paris

61 Avenue du Président Wilson, F-94235 Cachan Cedex, France E-mail: mathieu@lmt.enscachan.fr 
Keywords Covariance · Digital image correlation · Identification · Integrated approach $\cdot$ Ramberg-Osgood model

\section{Introduction}

Various identification methods have been developed through the last decades [6], some of them following the growing use of full-field measurements [26]. Finite Element Model Updating (or FEMU) is the most widely utilized identification technique $[34,29,42])$. It is usually an iterative method that relies on the comparison of the measured and computed loads and/or displacement fields $[34,17]$. However, in this method the measured displacement fields are treated as would be any point-wise measurement, namely, as data to post-process. In all the afore-mentioned cases, least squares errors are considered with no special emphasis on their weighting. Other quantities can also be considered, for instance by using "fields" of strain gages [33] or strain fields derived from measured displacement fields [37]. Other metrics have also been proposed when applied to the measured displacement or derived strain fields (e.g., the constitutive equation error $[15,22,1,21])$.

When dealing with elastic problems, various approaches rely on variational principles $[11,10]$. For instance, the Reciprocity Gap Method [3, 4]) only needs surface measurements to identify bulk defects. Non-iterative methods have also been developed, which make use of strain fields in elasticity (i.e., the Virtual Fields Method, or VFM [25, 43]) or measured displacement fields in elasticity and damage (i.e., the Equilibrium Gap Method [16, 48]). All these methods have been compared for elastic cases [6], where the noise/signal ratio is the main difficulty since the displacement and strain levels are very small.

Some of the previous methods have also been applied to the identification of elastoplastic parameters where the nonlinear dependencies between the pa- 
rameters may be difficult to handle. For instance, FEMU has been applied to the case of micro-structural deformations of grains in a metallic alloy [28], or at the mesoscopic level for quasi static [54] and dynamic [53] loading conditions. Avril et al. [7] discuss an example of plastic law identified for a tensile specimen with the VFM. Force/displacement FEMU has also been applied to different types of samples [44]. Cooreman et al. [19] show the advantage of calculating analytically the sensitivity matrix in these inverse methods.

It is worth noting that in all the studies listed so far, very few have explicitly dealt with measurement uncertainties. Conversely, optimized virtual fields have been constructed to account for noise sensitivity [5]. Similarly, minimizing the sensitivity to measurement uncertainties has enabled for the identifiability of load and contrast fields in microcantilevers [2]. Last, Gras et al. [23, 24] have proposed weighted least squares (or chi-squared) errors based upon the covariance matrix of measurement errors. This proposition will also be considered hereafter.

In all the listed identification techniques, the measured data are the input to the identification procedure. Therefore, the measurement step is performed prior to the identification step. An alternative route to these approaches is to resort to so-called integrated approaches in which the measurement and identification steps are performed in a single analysis. The measured (generalized) degrees of freedom then correspond to material parameters (e.g., elastic coefficients [30, 36], Paris' law parameters [40], damage parameters [46]), or mechanical parameters (e.g., stress intensity factors [47, 27, 38]). Most of the results obtained via Integrated-DIC (or I-DIC) have used closed-form solutions $[30,47,27,38]$ even though numerical solutions have also been considered $[36,46]$. The latter ones are more generic when dealing with nonlinear constitutive models. 
In the following, an integrated DIC code will be used to identify the elastoplastic parameters of Ramberg-Osgood's model [45]. The aim of the present study is not to analyze complex constitutive equations but rather to discuss the framework of DIC approaches in which the finite element code (here the commercial code Abaqus) are used in a non-intrusive way to generate the sensitivity fields for the identification procedure. Second, the results obtained with I-DIC will be compared with those utilizing FEMU in which a weighted least squares error is introduced. Last, the parameter resolutions are estimated by assessing the associated covariance matrix.

\section{Digital Image Correlation}

Digital Image Correlation is a widely used displacement field measurement technique $[52,32]$. It relies on the registration of an image $f$ in the reference configuration and a series of pictures $g$ in the deformed configurations. The registration operation consists of minimizing the sum of squared differences between the deformed image corrected for its displacement and the reference image. Many equivalent conventions can be chosen for the functional to be minimized, and this makes little or no difference for the determination of the displacement field. In particular, the scale for the objective functional is free and does not interfere any further.

In this paper, the choice is made to use this freedom to highlight the crucial role of noise in many applications of DIC. Successive acquisitions without any change in the conditions do not lead to identical images. They all differ from an inaccessible ideal image by a random noise that can only be described in a statistical sense. Although the latter is to be evaluated experimentally before and/or after the mechanical test, the specific case of white noise (i.e., uncorrelated from pixel to pixel) is chosen. Moreover, the probability distribution 
function is in many circumstances Gaussian [24]. In such cases, only one parameter characterizes the noise, namely its standard deviation $\gamma_{f}$ expressed in gray levels. There are ways to incorporate deviations from the above assumptions in different variational formulations of DIC [32] but the present study is restricted to this generic case of white Gaussian noise.

The noise amplitude sets a natural scale to measure the difference in gray levels between two images. Thus, it is proposed, at variance with standard practices, to define the objective functional to be minimized by the following intensive expression (i.e., quantity independent of the ROI area for a homogeneous texture)

$$
\eta_{t}^{2}(\mathbf{u})=\frac{1}{2 \gamma_{f}^{2}|\Omega|} \sum_{\Omega}(g(\mathbf{x}+\mathbf{u}(\mathbf{x}, t), t)-f(\mathbf{x}))^{2}
$$

This functional is to be minimized with respect to the parametrization of the sought displacement field $\mathbf{u}(\mathbf{x}, t)$, where $\mathbf{x}$ is any considered pixel, and $t$ the considered time. In this expression $\Omega$ designates the Region of Interest (ROI), $|\Omega|$ its area in terms of number of pixels it contains, and $\gamma_{f}$ the standard deviation of noise expressed in gray levels. Because noise affects both images $f$ and $g$, the pre-factor $1 /\left(2 \gamma_{f}^{2}|\Omega|\right)$ has been chosen in such a way that the mathematical expectation of the above functional solely due to noise is unity (i.e., when two consecutive images of the same scene are shot without any wanted difference).

Note that $\eta_{t}$ is independent of the gray level encoding. Even if this choice is not standard, it has no consequence on further results as the displacement that minimizes $\eta_{t}$ does not depend on the scale factor. An a priori displacement basis must be chosen for $\mathbf{u}(\mathbf{x}, t)$ to minimize $\eta_{t}$ with respect to the associated amplitudes. Continuous displacement fields found in finite element discretizations are one possible choice [14, 51, 8], which leads to lower uncertainties in the measured displacement fields [31], but more importantly can be used 
in finite element settings. Most of the applications reported so far deal with regular meshes made of 4-noded elements [51, 8, 48, 54, 53, 20]. However, unstructured meshes can also be used [14, 36, 55]. In the present analyses, three-noded (i.e., T3) elements will be considered.

The minimization of $\eta_{t}$ is solved by successive linearizations and corrections, using modified Newton's scheme [31]

$$
[\mathbf{M}]\left\{\delta \mathbf{u}_{\mathbf{m}}\right\}=\left\{\mathbf{b}^{(i)}\right\}
$$

where $[\mathbf{M}]$ is the DIC matrix, $\left\{\delta \mathbf{u}_{\mathbf{m}}\right\}$ the vector gathering all increments of measured displacement amplitudes, and $\left\{\mathbf{b}^{(i)}\right\}$ the residual column vector at iteration $i$, which cancels out when the gray level conservation is satisfied everywhere. When the system is invertible, the matrix $[\mathbf{M}]$ is symmetric, positive and definite. It can therefore be used to define a scalar product (i.e., $\left\{\mathbf{a}_{1}\right\} \bullet\left\{\mathbf{a}_{2}\right\}=\left\{\mathbf{a}_{1}\right\}^{t}[\mathbf{M}]\left\{\mathbf{a}_{2}\right\}$ ) and the corresponding $\mathbf{M}$-norm (i.e., $\left.\|\{\mathbf{a}\}\|_{\mathbf{M}}^{2}=\{\mathbf{a}\}[\mathbf{M}]\{\mathbf{a}\}\right)$. In a small neighborhood of the actual displacement field $\{\mathbf{u}\}$ separating the two images $\eta_{t}^{2}$ reads

$$
\eta_{t}^{2}(\{\mathbf{v}\}) \approx \frac{1}{2 \gamma_{f}^{2}|\Omega|}\|\{\mathbf{v}\}-\{\mathbf{u}\}\|_{\mathbf{M}}^{2}
$$

Since displacement measurements are corrupted by noise, the DIC matrix is also of importance when evaluating the covariance matrix $\left[\mathbf{C}_{\mathbf{u}}(t)\right]$ of the measured degrees of freedom $[8,31]$

$$
\left[\mathbf{C}_{\mathbf{u}}(t)\right]=2 \gamma_{f}^{2}[\mathbf{M}]^{-1} .
$$

The DIC code used herein is run independently for each picture pair. However, the registration of the $n$-th image pair is initialized with the measured displacement field of the $(n-1)$-th pair. It is worth noting that a spatiotemporal approach could also have been used [9].

In fact, it is possible to show that the use of the same reference image, itself affected by noise, leads to a non vanishing covariance for different image 
pairs as they share the same reference image

$$
\left[\mathbf{C}_{\mathbf{u}}\left(t, t^{\prime}\right)\right]=\gamma_{f}^{2}[\mathbf{M}]^{-1}
$$

for $t \neq t^{\prime}$. However, to limit the usage of memory in the following analyses these covariances will be neglected.

\section{Identification Methods}

Several identification methods have been adapted or developed to quantitatively use DIC results to identify material parameters $[6,26]$. The aim of the present work is to evaluate the performances of Integrated Digital Image Correlation (I-DIC) with respect to well accepted methods for the case of plasticity. The most used (i.e., reference) method (FEMU) is chosen herein and will be compared to I-DIC. However, the standard least squares setting will be substituted by a weighted approach considering the covariance matrix $\left[\underline{\mathbf{C}_{\mathbf{u}}}\right]$, which is block diagonal gathering all covariance matrices $\left[\mathbf{C}_{\mathbf{u}}(t)\right]$.

\subsection{Weighted Displacement-Based Finite Element Model Updating} (FEMU-U)

The displacement-based Finite Element Model Updating method was developed for using any measurement result to identify material parameters [17, 42]. In the present case, 2D measured displacement fields are considered as input data of such an identification procedure. The measured field $\mathbf{u}_{\mathbf{m}}$ is to be compared to the computed one $\mathbf{u}_{\mathbf{c}}$ by minimizing the weighted square norm (or chi-squared error)

$$
\chi_{u}^{2}(\{\mathbf{p}\})=\frac{1}{N_{u}}\left(\left\{\mathbf{u}_{\mathbf{m}}\right\}-\left\{\mathbf{u}_{\mathbf{c}}\right\}\right)^{t}\left[\underline{\mathbf{C}_{\mathbf{u}}}\right]^{-1}\left(\left\{\mathbf{u}_{\mathbf{m}}\right\}-\left\{\mathbf{u}_{\mathbf{c}}\right\}\right)
$$


where $\{\mathbf{p}\}$ is the column vector gathering all unknown material parameters needed for the computation of $\mathbf{u}_{\mathbf{c}}$. The normalization factor $1 / N_{u}$, where $N_{u}$ denotes the total number of kinematic degrees of freedom, has been introduced so that $\chi_{u}$ is an intensive quantity akin to $\eta$. In the present case, the covariance matrix $\left[\mathbf{C}_{\mathbf{u}}(t)\right]$ is equal to $2 \gamma_{f}^{2}[\mathbf{M}]^{-1}$ (see Equation (2)). The weighted chisquared error therefore refers to the M-norm

$$
\chi_{u}^{2}(\{\mathbf{p}\})=\frac{1}{2 \gamma_{f}^{2} N_{u}}\left\|\left\{\mathbf{u}_{\mathbf{m}}\right\}-\left\{\mathbf{u}_{\mathbf{c}}\right\}\right\|_{\mathbf{M}}^{2}
$$

It is important to note the analogy between the above expression and the previous DIC functional $\eta_{t}$, see Equation (3). Subsection 3.3 will discuss the rationale behind such a proximity.

The global minimum of $\chi_{u}^{2}$ corresponds to the best set of parameters $\{\mathbf{p}\}$ that can be found for the selected model. As for DIC, the minimization of $\chi_{u}^{2}$ is performed by successive linearizations and corrections. In the present case, the new estimate (i.e., at iteration $i$ ) of the computed displacement field reads

$$
\mathbf{u}_{\mathbf{c}}\left(\mathbf{x}, t,\left\{\mathbf{p}^{(i)}\right\}\right)=\mathbf{u}_{\mathbf{c}}\left(\mathbf{x}, t,\left\{\mathbf{p}^{(i-1)}\right\}\right)+\frac{\partial \mathbf{u}_{\mathbf{c}}}{\partial\{\mathbf{p}\}}\left(\mathbf{x}, t,\left\{\mathbf{p}^{(i-1)}\right\}\right)\{\delta \mathbf{p}\}
$$

where $\left\{\mathbf{p}^{(i-1)}\right\}$ is the set of parameters at iteration $i-1$, and $\{\delta \mathbf{p}\}$ the sought parameter increment. The computation of the sensitivity fields $\partial \mathbf{u}_{\mathbf{c}} / \partial\{\mathbf{p}\}$ can be performed analytically $[19,36]$ or numerically by computing the fields for small variations of each parameter of the considered set, as is the case herein by resorting to the commercial FE code Abaqus. In practice, forward finite differences are considered to compute the sensitivity fields. This set of sensitivity fields is computed for each iteration of the procedure. Once these fields are computed, the corrections to the sought parameters read

$$
\{\delta \mathbf{p}\}=\left[\mathbf{H}^{(i-1)}\right]^{-1}\left[\mathbf{S}_{\mathbf{u}}{ }^{(i-1)}\right]^{t}[\underline{\mathbf{M}}]\left(\left\{\mathbf{u}_{\mathbf{m}}\right\}-\left\{\mathbf{u}_{\mathbf{c}}{ }^{(i-1)}\right\}\right)
$$

where $\left[\mathbf{S}_{\mathbf{u}}{ }^{(i-1)}\right]$ is the matrix gathering all sensitivity fields (i.e., $\left\{\delta \mathbf{u}_{\mathbf{c}}\right\}=$ $\left[\mathbf{S}_{\mathbf{u}}\right]\{\delta \mathbf{p}\}$ ) (i.e., each raw gathers all nodal values of the sensitivity field for one 
of the considered parameter), $[\underline{\mathbf{M}}]$ the block diagonal matrix with the same matrix $[\mathbf{M}]$ for each considered time $t$, and $\left[\mathbf{H}^{(i-1)}\right]=\left[\mathbf{S}_{\mathbf{u}}{ }^{(i-1)}\right]^{t}[\underline{\mathbf{M}}]\left[\mathbf{S}_{\mathbf{u}}{ }^{(i-1)}\right]$ the approximated Hessian.

The covariance matrix of the identified material parameters $\left[\mathbf{C}_{\mathbf{p}}\right]$ is obtained by propagating the measurement uncertainties from the vector of measured kinematic degrees of freedom $\left\{\mathbf{u}_{\mathbf{m}}\right\}$ to the sought parameters $\{\mathbf{p}\}$

$$
\left[\mathbf{C}_{\mathbf{p}}\right]=[\mathbf{H}]^{-1}\left[\mathbf{S}_{\mathbf{u}}\right]^{t}[\underline{\mathbf{M}}]\left[\mathbf{C}_{\mathbf{u}}\right][\underline{\mathbf{M}}]\left[\mathbf{S}_{\mathbf{u}}\right][\mathbf{H}]^{-1}=2 \gamma_{f}^{2}[\mathbf{H}]^{-1}
$$

where $[\mathbf{H}]$ is the Hessian matrix at convergence of the identification procedure.

In practice, a part of the measurement result is selected as Dirichlet boundary conditions for the FE computations. Consequently, the restriction to these boundaries of the measured displacements, $\left\{\mathbf{u}_{\mathbf{m}}\right\}_{\mathrm{d} \Omega}$, is an input (see Figure 1). The FE description of the measured field allows the same mesh to be used for the measurement and computation, and thus to avoid any reprojection step between the measurement result and the computation mesh, which would possibly induce interpolation errors. This is feasible provided the measurement mesh is fine enough to ensure small computation errors. It will be assumed in the following that it is the case. Otherwise, a refining step of this mesh can be necessary [39].

Figure 1 summarizes the different steps of weighted FEMU-U to get the optimal set of parameters $\left\{\mathbf{p}_{\mathbf{o p t}}\right\}$ and the corresponding covariance matrix $\left[\mathbf{C}_{\mathbf{p}}\right]$. First, the in-house DIC code [36] provides the measured displacement field and the DIC matrix $[\mathbf{M}]$. Second the in-house code FEMU-U is run. It requires an initial set $\left\{\mathbf{p}_{\mathbf{0}}\right\}$ of the sought parameters. It can be noted that the commercial FE code is used in a non-intrusive way, namely the only information required for the identification step is the computed displacement field with the current estimate of the sought parameters $\{\mathbf{p}\}$. 
3.2 Integrated Digital Image Correlation (I-DIC)

Integrated digital image correlation (I-DIC) is a global DIC technique that relies on a specific choice for the measured displacement field. As the user possesses a priori knowledge on the sample behavior, it is possible to choose a better displacement space than the general FE form. The set of parameters $\{\mathbf{p}\}$ is generally chosen as the space for the degrees of freedom of the correlation problem. This method often yields results with less uncertainty than those post-processing measured data $[47,36]$, as it allows to perform the measurement and identification of the sought parameters in one step instead of two. This is particularly important in the case of small displacements, where the signal-to-noise ratio can make the identification problem difficult to handle (e.g., in elasticity $[30,36])$.

The present method also uses the sensitivity fields $\left[\mathbf{S}_{\mathbf{u}}\right]$ that are defined in Equation (9) if and only if the same discretization is used in both cases. The boundary conditions are the same in both analyses so that the finite element code can also be used in the I-DIC approach to provide the sensitivity fields. The registration problem then consists of solving a series of linear systems

$$
\left[\mathbf{N}^{(i)}\right]\{\delta \mathbf{p}\}=\left\{\mathbf{B}^{(i)}\right\}
$$

with $\left[\mathbf{N}^{(i)}\right]=\left[\mathbf{S}_{\mathbf{u}}{ }^{(i)}\right]^{t}[\underline{\mathbf{M}}]\left[\mathbf{S}_{\mathbf{u}}{ }^{(i)}\right]$, which is the projection of the DIC matrix onto the sensitivity fields, and $\left\{\mathbf{B}^{(i)}\right\}=\left[\mathbf{S}_{\mathbf{u}}^{(i)}\right]^{t}\left\{\underline{\mathbf{b}}^{(i)}\right\}$ the projection of the DIC vector $\left\{\underline{\mathbf{b}}^{(i)}\right\}$, which gathers all vectors $\left\{\mathbf{b}^{(i)}\right\}$ since a single spatiotemporal analysis is performed. It is worth noting that Equation (11) uses the same setting as that given in Equation (2), but expressed directly in terms of the sought parameters rather than the kinematic degrees of freedom of the DIC mesh. The only additional information is the sensitivity fields, which express the a priori knowledge on the constitutive behavior. Consequently, the generalized degrees of freedom only include material parameters. It is also conceivable 
to add boundary conditions to ensure the stability and to limit dependencies to these prescribed data. The price to pay is related to the computation of the sensitivity fields. However, they are also needed in the FEMU-U approach so that the computation time is equivalent for both approaches.

For the two global DIC approaches developed herein, the quality of the registration is first checked by the mean level $\eta$ of $\eta_{t}$ over the whole series of pictures. Further, since the corrected image in the deformed configuration $g(\mathbf{x}+\mathbf{u}(\mathbf{x}, t), t)$ is computed for each considered time $t$, it is also possible to see whether there are some local deviations in terms of registration quality by computing the correlation residuals $g(\mathbf{x}+\mathbf{u}(\mathbf{x}, t), t)-f(\mathbf{x})$.

Figure 1 summarizes the different steps of I-DIC to get the optimal set of parameters $\left\{\overline{\mathbf{p}}_{\mathbf{o p t}}\right\}$ and the corresponding covariance matrix $\left[\overline{\mathbf{C}}_{\mathbf{p}}\right]$. First, the in-house DIC code provides the measured displacement field and the DIC matrix $[\mathbf{M}]$. From the measured displacement field, only the displacements of the nodes corresponding to the kinematic boundary conditions, $\left\{\mathbf{u}_{\mathbf{m}}\right\}_{\mathrm{d} \Omega}$, are kept (see, for instance, Figure 2). Second the in-house I-DIC code is run. It also requires an initial set $\left\{\mathbf{p}_{\mathbf{0}}\right\}$ of the sought parameters. It can be noted that the commercial FE code is again used in a non-intrusive way.

\subsection{Relationship between I-DIC and Weighted FEMU-U}

Based on the above presentation, $[\mathbf{N}]=[\mathbf{H}]$ and hence it could be concluded that both procedures are strictly equivalent. This holds for the resulting evaluation of the parameter vector $\{\mathbf{p}\}$ and its covariance matrix. When measurements are performed via I-DIC, the covariance matrix $\left[\overline{\mathbf{C}}_{\mathbf{p}}\right]$ is evaluated as in the global DIC setting (see Equation (4)), as

$$
\left[\overline{\mathbf{C}}_{\mathbf{p}}\right]=2 \gamma_{f}^{2}[\mathbf{N}]^{-1}
$$


This identity comes from the fact that the DIC metric, $\|\ldots\|_{\mathbf{M}}$, is naturally transported in the FEMU-U identification procedure through the covariance of the kinematic variables. In the following, it will be shown that both approaches lead to comparable (although not strictly identical) results. Subtle differences originate from the approximation of the minimum of the registration residuals, $\eta^{2}$, as a harmonic potential (see Equation (3)). When considering the full DIC problem, it is highly nonlinear because of the generally non-regular image texture $f$. When noise can be considered as slight perturbations, it may be argued that the harmonic approximation is well-founded. However, for larger levels of noise, the harmonic approximation breaks down.

Moreover, without restrictions on the sought displacement fields, DIC is an ill-posed problem. It is the restriction of the trial displacement fields to a specific vector space that cures this ill-posedness. When more freedom is given to the displacement fields (e.g., when using very small finite-elements) the proximity of ill-posedness is felt as a degradation of the conditioning of the problem (i.e., smaller eigen-values in matrix $[\mathbf{M}]$ appear). The danger here is to miss the absolute minimum and get trapped in secondary minima, so that the measured displacements may reveal erroneous. In the FEMU-U method, the displacement covariance matrix is computed solely from the reference image, and hence it will not be affected by false convergence to a secondary minima.

A further consequence of poor conditioning/high noise is that large deviations of the displacement fields may occur. They in turn may require higher order developments of the identification functional itself. This effect is especially pronounced for nonlinear constitutive laws, and for instance large contrasts in tangent elastic moduli and/or with fine meshes needed to capture geometric details [36]. A systematic bias may result from these anharmonic effects. The example discussed in the following will display such a trend. 


\subsection{Use of Load Measurements}

Up to this point, the emphasis has been put on full-field displacement measurements. However, purely kinematic information is not able to provide an absolute measurement of say Young's modulus as no data set stress scales. In principle, dimensionless quantities can still be extracted from purely kinematic data such as the ratio of yield stress to Young's modulus, or Poisson's ratio. Yet, depending on the test and specimen geometry, constitutive parameters may show different ability for being estimated.

It is therefore very helpful to use the load data, which are generally available. From the FEMU-U or I-DIC-U approaches, the global load $F_{c}$ is calculated (at each state where a picture has been acquired) from a set of constitutive parameters and the experimentally measured boundary conditions. Independently, the same load is being measured $F_{m}$. It is generally impossible to prescribe strict equality $F_{c}=F_{m}$. The most natural writing, along the same philosophy as that presented for the proper handling of redundant kinematic data is to introduce an additional equilibrium gap function

$$
\chi_{F}^{2}(\{\mathbf{p}\})=\frac{1}{\gamma_{F}^{2} N_{F}}\left(F_{c}-F_{m}\right)^{2}
$$

where $\gamma_{F}$ is the standard resolution of the load measurement, and $N_{F}$ the total number of load data. Thus the expectation value of $\chi_{F}$ is unity when only noise is at play. The identification is then the result of minimizing

$$
\chi_{\mathrm{tot}}^{2}=(1-\omega) \chi_{u}^{2}+\omega \chi_{F}^{2}
$$

summed over all states where an image is captured (together with the measurement of the load). Here $0<\omega<1$ is a (dimensionless) weight.

One difficulty is to set the weight $\omega$ because it is assumed to balance data coming from different sources, as they are coupled in $\chi_{\text {tot. }}^{2}$. It is proposed to use uncertainty as the common gauge to assess each information. The unusual 
introduction of noise variance within the different functionals $\eta, \chi_{u}$ and $\chi_{F}$ was dictated by the aim of reaching an expectation value of unity for each of those quantities under the sole action of noise (i.e., ignoring all other sources of uncertainties or approximations). Thus, this principle naturally leads to the choice $\omega^{*}=1-\omega^{*}=1 / 2$, with which again the expectation of $\chi_{\text {tot }}$ is unity. It means that the credit given to any source of information is gauged by the uncertainty it provides. This choice can be founded more solidly by considering Bayesian approaches to inverse problems [49].

The introduction of this new functional requires sensitivity force vectors to be assessed for each considered parameter. Different choices will be made hereafter to highlight the contribution of different parameters:

- when $\omega=\omega^{*}=1 / 2$, the above balance between kinematic and static information is privileged, hence this choice will be referred to by the acronym UF,

- when $\omega=0$, the load measurements are disqualified and hence only DIC measurements will be used. The acronym for this case is $\mathrm{U}$,

- when $\omega=1$, the DIC measurement only comes into play through the boundary conditions used in the modeling. DIC is not taken into account for bulk measurements and free edges. Consequently, only gaps from global equilibrium are assessed. The acronym for this case is $\mathrm{F}$.

\section{Test-Case: Performance Evaluation}

To quantify the performances of FEMU and I-DIC for elastoplastic materials, a test case is proposed. A simple Ramberg-Osgood law [45] is to be identified. Its general expression is

$$
\epsilon_{i j}=\frac{1+\nu}{E} \sigma_{i j}-\frac{\nu}{E} \sigma_{k k} \delta_{i j}+\frac{3 A}{2 \sigma_{0}}\left(\frac{\sigma_{e q}}{\sigma_{0}}\right)^{n-1} s_{i j}
$$

where $\epsilon, \sigma$ and $s$ are the Hencky strain, Cauchy stress and deviatoric stress tensors used in the built-in Ramberg-Osgood law of the commercial code 
Abaqus [50], $\sigma_{e q}$ von Mises' equivalent stress, $\sigma_{0}$ and $n$ material parameters. Last, $A$ is a constant conventionally chosen to be $A=2 \times 10^{-3}$. When the Young's Modulus E and Poisson's ratio $\nu$ are known, only two parameters have to be determined, namely, $\sigma_{0}$ and $n$. It is to be emphasized that for large $n$ values, a very high contrast in tangent moduli is being set between the elastic and fully developed plastic regime. Such a pronounced nonlinearity (although representative of reality) is a severe difficulty to address.

To test the sensitivity of the proposed identification procedure to noise, a series of synthetic pictures in the deformed configuration is created from a real image in the reference configuration and computed displacements fields. The chosen reference image is that of the actual experimental case studied in Section 5.1. This image is artificially deformed with computed displacement fields $\mathbf{u}_{\mathbf{c}}(\mathbf{x}, t)$ using Ramberg-Osgood's behavior with chosen values of the constitutive parameters, $E=90 \mathrm{GPa}, \nu=0.34, \sigma_{0}=100 \mathrm{MPa}$ and $n=10$. Let $\Phi_{t}$ denote the transformation from the reference configuration to the deformed configuration at time $t$ (i.e., $\left.\Phi_{t}(\mathbf{x})=\mathbf{x}+\mathbf{u}(\mathbf{x}, t)\right)$. To get the series of pictures $g(\mathbf{x}, t)$, the gray level conservation relationship $f(\mathbf{x})=g\left(\Phi_{t}(\mathbf{x}), t\right)$ has to be inverted: $g(\mathbf{x}, t)=f\left(\Phi_{t}^{-1}(\mathbf{x})\right)$. The computed displacement field $\mathbf{u}_{\mathbf{c}}(\mathbf{x}, t)$ is first evaluated for each pixel $\mathbf{x}$ by using the FE interpolation functions. Consequently, the transformation $\Phi_{t}$ is evaluated at each considered position $\mathbf{x}$. By resorting to linear gray level interpolations, the inverse transformation $\Phi_{t}^{-1}$ is evaluated for each pixel $\mathbf{x}$. The boundary conditions are set with either measured displacements of the actual test or free edge as appropriate (Figure 2). For the sake of computation time, this test case consists of the first 35 steps of the actual test. The total load is also computed for each analyzed image, and the force measurement uncertainty is set to the experimental level of Section 5 . 
Once the artificial image series has been built, noise can be added. In the present case Gaussian white noise is considered whose standard deviation $\gamma_{f}$ is varied. In the following, the effect of the noise level is investigated to evaluate its influence on the identification result, and assess the robustness of the identification procedure. Two values of $\gamma_{f}$ are chosen, namely, $0.6 \%$ and $3 \%$ of the dynamic range (i.e., difference between maximum and minimum gray levels) of the reference picture. The first value, $0.6 \%$, corresponds to typical levels for optical images [32]. The second value is high and is chosen to challenge robustness as large noise amplitudes could induce violations of the harmonic approximations used in many instances and the gray level conservation.

I-DIC and FEMU did not reveal significant differences, and hence only I-DIC is used in the following illustration. However, the relative weight of the load information with respect to the kinematic one was varied. As earlier mentioned, the three cases, $\omega=0, \omega=1 / 2$ and $\omega=1$ will be considered and referred to as U, UF and F respectively.

The identification results are presented for $n$ and $\sigma_{0}$ as functions of the number of images used in the analysis in Figure 3. The two noise levels are shown in different graphs. It is observed that for the standard $0.6 \%$ noise level, very fast and accurate determinations of the exponent $n$ are obtained for both I-DIC-U and I-DIC-F limits, and hence also for the UF case. For the same parameter $n$, the large noise case reduces the rate of convergence to the known value $n=10$, yet both $\mathrm{U}$ and $\mathrm{F}$ cases tend to the correct value as the number of analyzed pictures increases.

Considering the $\sigma_{0}$ parameter, it is seen that for both noise levels, the IDIC-U procedure leads to systematic underestimations (the same is true for FEMU-U). This is due to the very high contrast between the elastic and the plastic behavior and the geometry of the test. This result is explained by the fact that the reaction forces of the reference $\mathrm{FE}$ computation are not matched 
using the I-DIC-U procedure (see Figure 4). With the sole kinematic data (i.e., case $\mathrm{U}$ ), only the ratio $\sigma_{0} / E$ can be determined, and the rapid cross-over between the elastic and plastic regimes is such that most data are dominated by either $\sigma_{0}$ or $E$, and very few elements allow to rank one with respect to the other if no load information is given. As soon as the load information (i.e., $\mathrm{F}$ ) is given, both $\mathrm{F}$ and UF procedures lead to correct values for $\sigma_{0}$. For the standard noise level, the known value is reached for a very small number of analyzed pictures and does not depart from this expected estimate. For large noise levels, convergence is much slower, but for 35 analyzed pictures, the error in the evaluation of $\sigma_{0}$ is only about $3 \%$ and $5 \%$ for $n$, which is deemed very satisfactory given the high level of noise.

The correlation residual $\eta_{t}$ defined in Equation (1) is the natural tool to evaluate the registration quality of DIC approaches. Figure 5 shows the change of the mean residuals for the identified model parameters for the two noise levels. It is observed that all methods U, F and UF, give indistinguishable results. With the chosen normalization, high levels of noise lead to residuals of the order of unity (Figure 5(a)). Conversely, for very low levels they may depart from 1 (Figure 5(a)). To understand this effect, Figure 6 shows the level of dimensionless residuals with respect to the dynamic range $\Delta f$ of the ROI in the reference picture $f$

$$
\tilde{\eta}=\eta \frac{\sqrt{2} \gamma_{f}}{\Delta f}
$$

plotted for the different levels of noise, which are represented by straight lines. For low and zero image noise levels, gray level interpolations become an important source of error whereas for larger noise levels they become negligible. The large noise case shows quasi-constant $\eta$ values close to unity. This is typical of residuals mainly influenced by noise, and not by modeling errors or gray level interpolations. 
In all cases, a strong correlation between the two parameters $\sigma_{0}$ and $n$ is observed. This is consistent with the covariance matrix that can be estimated from the previous analysis,

$$
\left[\overline{\mathbf{C}}_{\mathbf{p}}\right]=\left(\begin{array}{cc}
0.16 & 0.43 \\
0.43 & 1.4
\end{array}\right)
$$

when $\{\mathbf{p}\}=\left\{\begin{array}{ll}n & \sigma_{0}\end{array}\right\}^{t}$. The corresponding correlation matrix reads

$$
[\mathbf{R}]=\left(\begin{array}{cc}
1 & 0.89 \\
0.89 & 1
\end{array}\right)
$$

where (no index summation used)

$$
R_{i j}=\frac{\left(\bar{C}_{p}\right)_{i j}}{\sqrt{\left(\bar{C}_{p}\right)_{i i}\left(\bar{C}_{p}\right)_{j j}}}
$$

To further analyze this effect, the sensitivity of the two residuals, namely, correlation residual $\eta$ (Equation (1)) and load residual $\chi_{F}$ (Equation (13)) are evaluated in the vicinity of the reference solution. More precisely, for the chosen range of $n$ and $\sigma_{0}$, the two residuals (DIC and global equilibrium) are computed. Figure 7(a) illustrates the previous result, namely, there is a very shallow valley along one direction in the $\left(\sigma_{0}, n\right)$ plane when the mean correlation residual alone is considered. Conversely, the equilibrium residual, Figure 7(b), is very sensitive to $\sigma_{0}$ and less to $n$. Let us stress the difference in scales for both functionals. The levels can be compared as 1 would correspond to the effect of noise in both cases. This trend explains the reason for the better estimation of $\sigma_{0}$ than $n$ in the presence of very severe noise levels.

Last, the computation time is nearly identical for both methods, for the same number of iterations. The most expensive operations being the FE computations of the sensitivity fields (Figure 1), the time for the construction of the global matrix is negligible in the present case. To compute all the results 
presented in this test case, around 15 hours have been necessary on a regular PC (i.e., from 2 to 40 minutes per analysis depending on the number of analyzed pictures).

\section{Analysis of an Experiment}

The sample geometry being simple, 40-pixel elements are used as in the test case (Figure 2). This is not always possible (i.e., when the geometry of the sample is more complex than in the present case [36]). The true images are processed for the identification of elastic and plastic parameters.

\subsection{Test Configuration}

A tensile test is performed on dog-bone sample made of commercially pure (T35) titanium. The region of interest is approximately $100 \mathrm{~mm}$ long, its ligament is $8 \mathrm{~mm}$ wide, and the sample is $0.3 \mathrm{~mm}$ thick. The reference image is shown in Figure 2. An 8-bit camera (definition: $2320 \times 1728$ pixels) is used with telecentric lens. The speckle is applied onto the sample with an ink-jet printer.

Unloading/reloading sequences are used for the identification of the Poisson's ratio $\nu$ and Young's modulus $E$, and the 'plastic' loading for the identification of the Ramberg-Osgood parameters $\sigma_{0}$ and $n$. The load level and displacement fields are stored for each analyzed image. Figure 8 indicates which parts of the loading history are selected for the identification. 
5.2 Elastic Unloading/Reloading Sequences

Ten unloading/reloading sequences are processed for the evaluation of the elastic parameters. In an unloading/reloading sequence, both loading directions are treated the same way, the images being sorted by load amplitude only.

The first parameter to determine is the Young's modulus. This is possible only if the load information is considered either during the identification per se, or subsequently when I-DIC-U is performed. Figure 9 shows that a stable value is reached very quickly with reasonable small fluctuations when the number of analyzed pictures is greater than 5 . The mean value of $E=90 \mathrm{GPa}$ is in good agreement with reported levels (i.e., $102 \mathrm{GPa}$ [13]), considering the fact that the sample is machined from $0.3-\mathrm{mm}$ thick rolled sheets.

For the Poisson's ratio, the (plane stress) results show a rather stable value close to the incompressible limit, $\nu=1 / 2$. Note however that this parameter is not allowed to go beyond the upper limit of $1 / 2$, as this is a strict thermodynamic constraint. This result is in conflict with the classically accepted value [18] of 0.34 . A closer inspection shows that this is not due to a lack of accuracy. Three independent codes (i.e., the one developed herein in addition to two others $[36,24])$ have been used for this determination and they all provided the same estimate. A difference of about 0.15 in Poisson's ratio is expected to give rise to a maximum transverse displacement of the order of 0.02 pixel (or $500 \mathrm{~nm}$ ). This difference albeit small can be resolved by the DIC procedure. However, because the specimen is very thin (i.e., $0.3 \mathrm{~mm}$ ), an axial rotation proportional to the load cannot be excluded, and about $10^{-2}$ rad-rotation is sufficient over the load increment in the elastic domain to account for such an offset in the Poisson's ratio. This rotation was not anticipated, and hence not prevented nor measured. Consequently the fact that transverse displacements cannot be considered as reliable forbids to give credit to the elastic 
Poisson's ratio. However, it should be noted that the axial displacement is much more significant than the transverse one (because of axial loading and elongated geometry), and there is no ambiguity about isochoric strains in the plastic regime. Thus, the disqualification of the Poisson's ratio does not lead to negative consequences for further identification of the other parameters.

To determine a possible signature of damage with respect to the number of unloading/reloading sequences, Figure 10 shows the identified values for a series of 15 images for each sequence. A progressive decrease for the larger strain levels suggests the relevance of damage effects. The uncertainty for $E$ being directly linked to that of the measured force, it is estimated of the order of $0.3 \mathrm{GPa}$ so that the degradation of stiffness is considered as real. Yet it only affects the last load levels. At most a 5\% decrease in the Young's modulus is observed at the very end of the test. Thus, in spite of these observations, this effect is discarded from the analysis to preserve the low number of parameters of the model.

\subsection{Plastic behavior}

One hundred images of the yielding material are used to determine the Ramberg-Osgood coefficients. The unloading and reloading cycles are excluded from this analysis. To estimate the influence of the chosen procedures, the three (U, F and UF) variants are run with the two proposed identification methods (FEMU and I-DIC).

Of the three variants, only U leads to slight differences in the results between FEMU and I-DIC. The results are presented in Figures 11 to 14 with respect to the number of images used in the $\mathrm{U}$ analyses. As for the test-case, the results are similar for both methods in terms of correlation residuals (Figure 11). One interesting feature is to observe the drift of the mean correlation residuals 
with the number of analyzed pictures. The typical level is at least three times that of noise. These results are clear indications that a model error occurs. In contrast with the artificial case that was treated in Section 4 and where the constitutive law was prescribed, it is very likely that the simple algebraic expression of Ramberg-Osgood law has too little freedom to precisely account for the entire data set. More advanced models can be considered (e.g., Ludwig's or Voce's propositions if isotropic hardening is still considered).

Figures 12 and 13 show the changes of the identified exponent $n$ and stress parameter $\sigma_{0}$. Similar trends are observed for the two methods and both parameters. The standard deviations are systematically lower for the integrated approach compared with FEMU. Further, the strong cross-correlation between the two parameters that is observed in Figure 14 makes their identification very delicate. Last, the identified values of $\sigma_{0}$ are much lower than expected (from classical stress/strain curve analyses that yield levels from 150 to $200 \mathrm{MPa}$ ).

As expected from the test-case, the consideration of the load information in the global functional is necessary to match the measured load with the computed reaction forces. The influence of each variant (U, F or UF) is shown in Figures 15 to 18 . It is chosen to present results only for I-DIC, as they are very similar for FEMU. Figure 15 shows that the mean correlation residual is only slightly degraded by the inclusion of the load information in the identification procedure. As in the previous analyses, its drift with the number of analyzed pictures leading to a two-fold increase is an indication that the chosen model is not able to properly describe the experimental results.

This result is confirmed when the dimensionless residual $\tilde{\eta}$ is compared with the results obtained in the previous section (Figure 16). The residuals for the real experiment are drifting when the number of analyzed pictures is increased thereby indicating that image noise is no longer the only influential factor so that the chosen model is not suited to the studied experiment. The 
gray level interpolations are not believed to be the cause of drift since the residual levels are too high.

Figure 17 shows that the use of the load measurement allows $\sigma_{0}$ to reach levels that are closer to the usual estimations of this parameter, and with lower resolutions. Remarkably, the change of $\sigma_{0}$ with the number of pictures is significantly lower in the present case when compared to the U variant (Figure 13). Further, as the number of pictures increases, the evaluation of the yield stress $\sigma_{0}$ become very close for the UF and $\mathrm{F}$ variants. The change of the identified power $n$ with the number of pictures is shown in Figure 18. Its variation is completely different when the equilibrium gap is considered or not. These similar trends observed for $n$ and $\sigma_{0}$ can be explained by the map of Figure 7(a) and the corresponding strong cross-correlations, which are only counter-balanced by the load information (Figure $7(\mathrm{~b})$ ).

When analyzing the measured and computed load levels (Figure 19), it is concluded that the correlation residuals alone are not sufficient to ensure the quality of the identified result. In the present case, the global equilibrium gap has to be considered as well. However, the fact that the correlation residuals experience significant deviations from the value corresponding to noise only is an indication that Ramberg-Osgood's model is a crude approximation of the behavior of the studied material.

Figures 15 to 18 show different results for procedures $F$ and UF. With the chosen weight $\omega=\omega^{*}=1 / 2$, the design of the test and the quality of the load measurement leads to different influences of $\eta$ and $\chi_{F}$ in the identification results as shown in Figure 20 when the standard deviations of the identified parameters with respect to noise corresponding to the two contributions are plotted. 


\section{Conclusion}

The present study aimed at showing that when the proper metric is considered in FEMU identification procedures, the result should ideally coincide with IDIC. However, that coincidence is limited to some conditions to be fulfilled, such as small noise amplitudes so that a harmonic approximation for the DIC objective functional is appropriate, and good conditioning of the DIC matrix. When coupled with identification, it is also necessary that the sought constitutive law accounts well for the actual displacement field.

It has been shown that I-DIC could be validated for artificial test cases where the constitutive parameters of Ramberg-Osgood's law could be retrieved accurately. The same procedure has been applied to real experimental data, and the elastic parameters and Ramberg-Osgood parameters have been evaluated. Over a large range of strains (i.e., up to $18 \%$ ), the displacement field determined from the identified constitutive law has led to DIC residual levels greater than 5 times that corresponding to acquisition noise. This comparison constitutes a way of assessing the model error in comparison with measurement errors associated with noise.

An important point to note is that the identification can be carried out based on computed displacement bases from the non intrusive use of (commercial) finite element softwares. Such coupling has already been proposed [12] for digital volume correlation based on elastic computations. Here an extension to elasto-plastic behaviors is considered in two dimensions. Such a procedure opens up new pathways, where one can benefit from the almost unlimited capabilities of nowadays FE softwares coupled with 2D-DIC, stereoDIC or DVC.

Last, the main aim of the present analyses was to validate the I-DIC procedures against more classical identification techniques (i.e., FEMU in the present case). Being validated, more complex meshes and geometries, which 
require a significant decrease of the spatial resolution, will be considered. It was already shown that standard DIC analyses become less secure or can even diverge when aiming for meshes at the scales of the pixels [36, 41, 55]. The subsequent identification breaks down even when DIC results can be used [36]. Conversely, regularized and integrated DIC can be performed at such fine resolutions and still yield reliable displacement fields $[41,55]$ and material parameters [36]. Such route constitutes one of the next steps to be investigated. This is particularly important when investigating localized phenomena associated with plasticity $[54,53,35]$.

\section{Acknowledgments}

It is a pleasure to acknowledge the support of Région Ile de France ("FRESCORT" and "DICCIT" projects).

\section{References}

1. Allix O, Feissel P, Nguyen H (2005) Identification strategy in the presence of corrupted measurements. Eng Comput 22(5-6):487-504

2. Amiot F, Hild F, Roger J (2007) Identification of elastic property and loading fields from full-field displacement measurements. Int J Solids Struct 44:2863-2887

3. Andrieux S, Abda AB, Bui H (1999) Reciprocity principle and crack identification. Inv Problems 15:59-65

4. Andrieux S, Bui H, Constantinescu A (2012) Reciprocity Gap Method. In: Grédiac M, Hild F (edts.) Full-Field Measurements and Identification in Solid Mechanics. ISTE / Wiley, London (UK), pp 363-378

5. Avril S, Grédiac M, Pierron F (2004) Sensitivity of the virtual fields method to noisy data. Comput Mech 34(6):439-452 
6. Avril S, Bonnet M, Bretelle A, Grédiac M, Hild F, Ienny P, Latourte F, Lemosse D, Pagano S, Pagnacco E, Pierron F (2008) Overview of identification methods of mechanical parameters based on full-field measurements. Exp Mech 48(4):381-402

7. Avril S, Pierron F, Pannier Y, Rotinat R (2008) Stress reconstruction and constitutive parameter identification in plane-stress elasto-plastic problems using surface measurements of deformation fields. Exp Mech 48(4):403-419

8. Besnard G, Hild F, Roux S (2006) "finite-element" displacement fields analysis from digital images: Application to Portevin-le Chatelier bands. Exp Mech 46:789-803

9. Besnard G, Leclerc H, Roux S, Hild F (2012) Analysis of Image Series through Digital Image Correlation. J Strain Analysis 47:214-228

10. Bonnet M (2012) Introduction to Identification Methods. In: Grédiac M, Hild F (edts.) Full-Field Measurements and Identification in Solid Mechanics. ISTE / Wiley, London (UK), pp 223-246

11. Bonnet M, Constantinescu A (2005) Inverse problems in elasticity. Inv Problems 21:R1-R50

12. Bouterf A, Roux S, Hild F, Adrien J, Maire E (2014) Digital volume correlation applied to x-ray tomography images from spherical indentation tests on lightweight gypsum. Submitted for publication

13. Boyer R, Welsch G and Collings E W (1994) Materials Properties Handbook: Titanium Alloys, eds. ASM International

14. Broggiato G (2004) Adaptive image correlation technique for full-field strain measurement. In: Pappalettere C (edt.) 12th Int Conf Exp Mech. McGraw Hill, Bari (Italy), pp 420-421

15. Calloch S, Dureisseix D, Hild F (2002) Identification de modèles de comportement de matériaux solides : utilisation d'essais et de calculs. Technol 
Formations 100:36-41

16. Claire D, Hild F, Roux S (2004) A finite element formulation to identify damage fields: The equilibrium gap method. Int J Num Meth Engng 61(2):189-208

17. Collins J, Hart G, Kennedy B (1974) Statistical identification of structures. AIAA J 12(2):185-190

18. Conrad H, Jones R (1970) The Science, Technology and Application of Titanium. Pergamon Press, Oxford (UK)

19. Cooreman S, Lecompte D, Sol H, Vantomme J, Debruyne D (2007) Elastoplastic material parameter identification by inverse methods: Calculation of the sensitivity matrix. Int J Solids Struct 44(13):4329-4341

20. Fagerholt E, Børvik T, Hopperstad OS (2013) Measuring discontinuous displacement fields in cracked specimens using digital image correlation with mesh adaptation and crack-path optimization. Optics and Lasers in Engineering 51(3):299-310

21. Feissel P, Allix O (2007) Modified constitutive relation error identification strategy for transient dynamics with corrupted data: The elastic case. Comput Meth Appl Mech Eng 196(13/16):1968-1983

22. Geymonat G, Hild F, Pagano S (2002) Identification of elastic parameters by displacement field measurement. C R Mécanique 330:403-408

23. Gras R, Leclerc H, Roux S, Otin S, Schneider J, Périé J (2013) Identification of the out-of-plane shear modulus of a $3 d$ woven composite. Exp Mech 53:719-730

24. Gras R, Leclerc H, Hild F, Roux S, Schneider J (2013) Identification of a set of macroscopic elastic parameters in a $3 \mathrm{~d}$ woven composite: Uncertainty analysis and regularization. Int. J. Solids Struct., DOI: 10.1016/j.ijsolstr.2013.12.023 
25. Grédiac M (1989) Principe des travaux virtuels et identification. C R Acad Sci Paris 309(Série II):1-5

26. Grédiac M, Hild F, edts (2012) Full-Field Measurements and Identification in Solid Mechanics. ISTE / Wiley, London (UK)

27. Hamam R, Hild F, Roux S (2007) Stress intensity factor gauging by digital image correlation: Application in cyclic fatigue. Strain 43:181-192

28. Héripré E, Dexet M, Crépin J, Gélébart L, Roos A, Bornert M, Caldemaison D (2007) Coupling between experimental measurements and polycrystal finite element calculations for micromechanical study of metallic materials. Int J Plasticity 23(9):1512-1539

29. Hermez F, Farhat C (1993) Updating finite element dynamic models using element-by-element sensitivity methodology. AIAA J 31(9):1702-1711

30. Hild F, Roux S (2006) Digital image correlation: From measurement to identification of elastic properties - A revision. Strain 42:69-80

31. Hild F, Roux S (2012) Comparison of local and global approaches to digital image correlation. Exp Mech 52(9):1503-1519

32. Hild F, Roux S (2012) Digital Image Correlation. In: Rastogi P, Hack E, edts. (2012) Optical Methods for Solid Mechanics. A Full-Field Approach. Wiley-VCH, Weinheim (Germany), pp 183-228

33. Kavanagh K (1972) Extension of classical experimental techniques for characterizing the composite-material behavior. Exp Mech 12(1):50-56

34. Kavanagh K, Clough R (1971) Finite element applications in the characterization of elastic solids. Int J Solids Struct 7:11-23

35. Kim J-H, Serpantié A, Barlat F, Pierron F, Lee M-G (2013) Characterization of the post-necking strain hardening behavior using the virtual fields method. Int. J. Solids Struct. 50:3829-3842

36. Leclerc H, Périé J, Roux S, Hild F (2009) Integrated digital image correlation for the identification of mechanical properties, LNCS, Springer, 
Berlin, 5496:161-171

37. Lecompte D, Smits A, Sol H, Vantomme J, Hemelrijck D (2007) Mixed numerical-experimental technique for orthotropic parameter identification using biaxial tensile tests on cruciform specimens. Int J Solids Struct 44(5):1643-1656

38. Mathieu F, Hild F, Roux S (2012) Identification of a crack propagation law by digital image correlation. Int J Fat 36:146-154

39. Mathieu F, Aimedieu P, Guimard J, Hild F (2013) Identication of interlaminar fracture properties of a composite laminate using local full-field kinematic measurements and finite element simulations. Comp Part A 49:203-213

40. Mathieu F, Hild F, Roux S (2013) Image-based identification procedure of a crack propagation law. Eng Fract Mech 103:48-59

41. Passieux JC, Périé JN (2012) Digital image correlation using proper generalized decomposition: PGD-DIC. Int. J. Num. Meth. Eng. 92:531-550

42. Pagnacco E, Caro-Bretelle A, Ienny P (2012) Parameter Identification from Mechanical Field Measurements using Finite Element Model Updating Strategies. In: Grédiac M, Hild F, edts (2012) Full-Field Measurements and Identification in Solid Mechanics. ISTE / Wiley, London (UK), pp $247-274$

43. Pierron F, Grédiac M (2012) The Virtual Fields Method. Springer

44. Pottier T, Toussaint F, Vacher P (2011) Contribution of heterogeneous strain field measurements and boundary conditions modelling in inverse identification of material parameters. Eur J Mech A/Solids 30(3):373-382

45. Ramberg W, Osgood WR (1943) Description of stress-strain curves by three parameters. Tech. rep., National Advisory Committee For Aeronautics, Washington DC (USA) 
46. Réthoré J (2010) A fully integrated noise robust strategy for the identification of constitutive laws from digital images. Int J Num Meth Eng $84(6): 631-660$

47. Roux S, Hild F (2006) Stress intensity factor measurements from digital image correlation: post-processing and integrated approaches. Int J Fract 140(1-4):141-157

48. Roux S, Hild F (2008) Digital image mechanical identification (DIMI). Exp Mech 48(4):495-508

49. Simoncelli EP (1999) Bayesian Multi-Scale Differential Optical Flow. In: Jähne B, Haussecker H, Geissler P (eds) Handbook of Computer Vision and Applications, vol 2. Academic Press, pp 297-422

50. Simulia (2009) Abaqus Analysis User's Manual, 19.1.1. Inelastic behavior. Dassault Systèmes, Providence, RI (USA)

51. Sun Y, Pang J, Wong C, Su F (2005) Finite-element formulation for a digital image correlation method. Appl Optics 44(34):7357-7363

52. Sutton M, Orteu J, Schreier H (2009) Image correlation for shape, motion and deformation measurements: Basic Concepts, Theory and Applications. Springer, New York, NY (USA)

53. Tarigopula V, Hopperstad O, Langseth M, Clausen A, Hild F (2008) A study of localisation in dual phase high-strength steels under dynamic loading using digital image correlation and fe analysis. Int J Solids Struct $45(2): 601-619$

54. Tarigopula V, Hopperstad O, Langseth M, Clausen A, Hild F, Lademo O, Eriksson M (2008) A study of large plastic deformations in dual phase steel using digital image correlation and fe analysis. Exp Mech 48(2):181-196

55. Tomicevic Z, Hild F, Roux S (2013) Mechanics-aided digital image correlation. J Strain Analysis 48:330-343 


\section{List of Figures}

1 Flowchart showing the different steps of weighted FEMU-U (left) and I-DIC-U (right). Both approaches need different results of a first DIC analysis, where $\left\{\mathbf{u}_{\mathbf{m}}\right\}_{d \Omega}$ denotes the restriction of the measured displacement displacements to the nodes where kinematic boundary conditions are prescribed, and FE

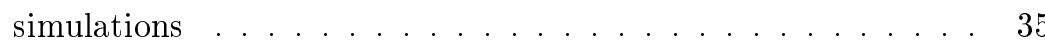

2 Horizontal displacement for image 4 (expressed in pixels, whose physical size is $27 \mu \mathrm{m})$. The green points indicate the nodes where displacements are prescribed in the FE computations. The remainder of the boundary is free. The notch radius is equal to $100 \mathrm{~mm}$, and the ligament width $8 \mathrm{~mm} \ldots \ldots$. . .

3 Identified parameters $n$ (top row (a) and (b)) and $\sigma_{0}$ in $\mathrm{MPa}$ (bottom row (c) and (d)). The left graphs ((a) and (c)) correspond to standard noise level $(0.6 \%$ of the dynamic range of $f$ ). The right graphs ((b) and (d)) show the corresponding identified values for a high noise level (3\% of the dynamic range of $f) \ldots \ldots \ldots \ldots \ldots \ldots$

4 (a) Elongation history of the experiment reported hereafter. The blue circles are used as input for the test case. (b) Computed sum of the reaction forces of the numerical specimen for the reference computation, for the I-DIC-U and I-DIC-UF procedures. The reaction forces are computed using the parameters identified with 35 images. The use of $\chi_{F}^{2}$ is necessary to match the reference reaction forces. The load fluctuations are due to the fact that actual displacements are prescribed on the two boundaries shown in Figure $2 \ldots \ldots . \ldots . \ldots 38$ 
5 Correlation residuals $\eta$ obtained for the identified parameters using the three U, F and UF procedures. The left graph (a) corresponds to a standard noise level $(0.6 \%$ of the dynamic range of $f$ ) and the right graph (b) to a large noise level (3\% of the dynamic range of $f$ ) . . . . . . . . . . .

6 Dimensionless correlation residual $\tilde{\eta}$ for the test-case for different noise levels. As the identified model is that used to deform the pictures, the error sources are image noise and gray level interpolations. For zero and low noise levels, the gray level interpolation error is predominant .............

$7 \quad$ Correlation residual $\eta$ (a) and load residual $\chi_{F}$ (b) as functions of the value of the plastic parameters $\sigma_{0}$ and $n$ when the reference values are equal to $100 \mathrm{MPa}$ and 10, respectively, and $0.6 \%$ noise is applied . . . . . . . . . . .

8 Plot of the load/displacement curve. The red zones indicate the parts where images are selected for the determination of elastic parameters, the blue parts for plastic parameters . . . . . . .

9 Identified Young's modulus for 10 unloading/reloading sequences. Each point represents an evaluation of $E$ based on the two proposed identification methods and using different numbers of images in each sequence . . . . . . . . . . . .

10 Identified Young's moduli, for 15 images, for each of the 10 unloading/reloading sequences. A slight decrease may be observed and may be attributed to damage. However, because of the small amplitude of decrease, damage will not be considered

11 Correlation residual $\eta$ after convergence of the algorithms. For FEMU-U, the level is an equivalent residual computed for one iteration of the I-DIC procedure after its convergence . . . . . . 4 
12 Identification results for Ramberg-Osgood parameter $n$, and corresponding standard deviation due to noise. There are few differences between the results of both methods, except with a large number of images . . . . . . . . . . . . . 45

13 Identification results for Ramberg-Osgood parameter $\sigma_{0}$, and corresponding resolution. The standard deviation due to noise being directly dependent on the parameter level, the inverse shape of the $\sigma_{0}$ curve can be observed in the standard deviation 46

14 Cross-correlation of the $\left(n, \sigma_{0}\right)$ pair. As in the test-case, its level is very high (around 0.9) and influences the identification of both parameters .................... 47

15 Correlation residual $\eta$ after convergence of the algorithms. The residual is very similar with or without the use of load information 48

16 Dimensionless correlation residuals $\tilde{\eta}$ for the test-case and the actual experiment. The results for the actual experiment show a significant drift that is not to be expected for this level of noise except if a model error arises . . . . . . . . . . . . . 48

17 Identification results for parameter $\sigma_{0}$, and corresponding resolution. The use of the equilibrium gap functional $\chi_{F}$ allows a steadier value of $\sigma_{0}$ to be identified and a lower standard reso-

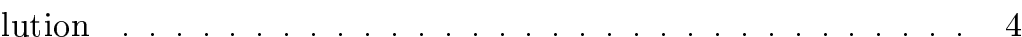

18 Identification results for parameter $n$, and corresponding resolution. The use of the force function $\chi_{F}$ changes significantly the identified values of $n$ but not its standard resolution . . . 50 
19 Measured force and computed sum of the reaction forces for the I-DIC-U and I-DIC-UF procedures. The reaction forces are computed using the parameters identified with 90 images. This result shows that using only $\eta$ can lead to poor results in the

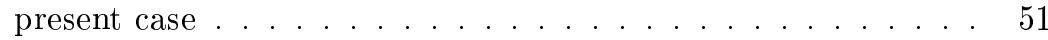

20 Standard resolutions derived from the covariance matrices of $\eta$ and $\chi_{F}$ for parameters $n$ and $\sigma_{0} \ldots \ldots \ldots \ldots 2$ 


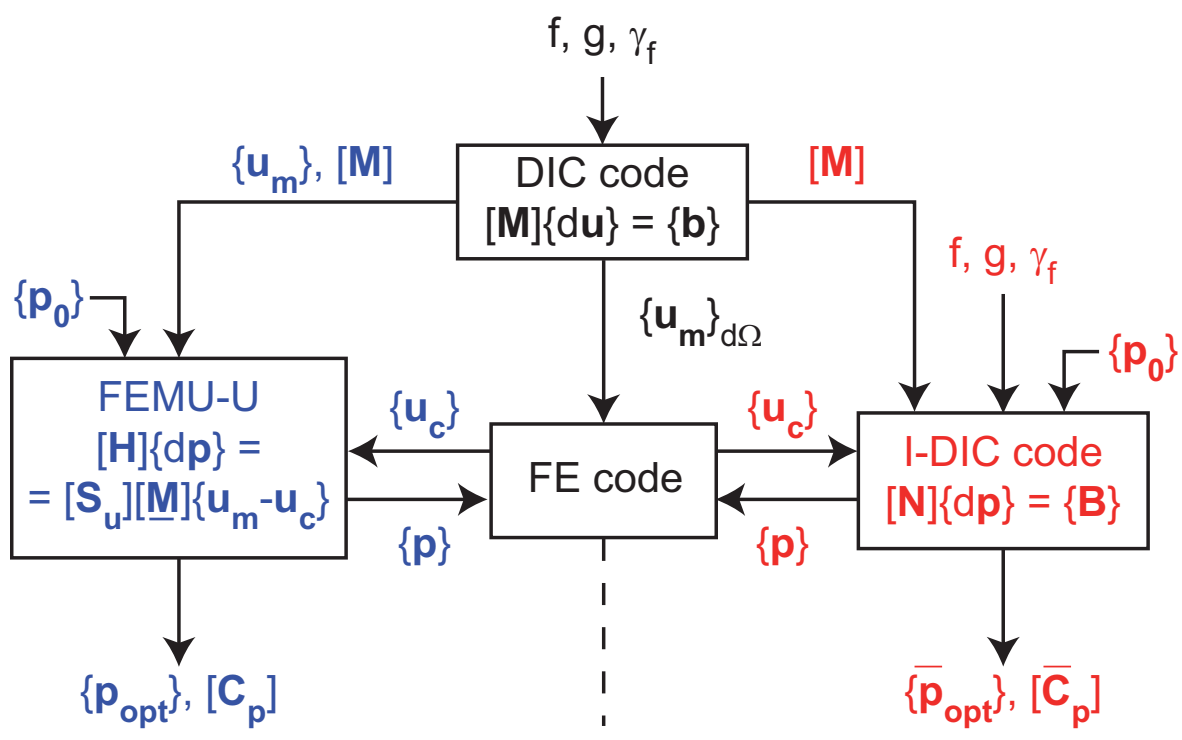

Fig. 1 Flowchart showing the different steps of weighted FEMU-U (left) and I-DIC-U (right). Both approaches need different results of a first DIC analysis, where $\left\{\mathbf{u}_{\mathbf{m}}\right\}_{d \Omega}$ denotes the restriction of the measured displacement displacements to the nodes where kinematic boundary conditions are prescribed, and FE simulations 


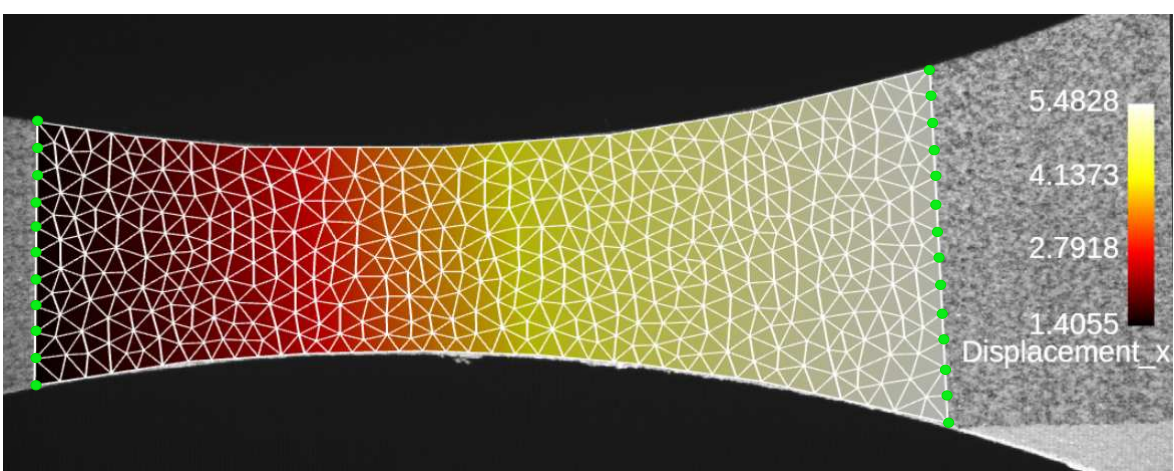

Fig. 2 Horizontal displacement for image 4 (expressed in pixels, whose physical size is $27 \mu \mathrm{m})$. The green points indicate the nodes where displacements are prescribed in the FE computations. The remainder of the boundary is free. The notch radius is equal to $100 \mathrm{~mm}$, and the ligament width $8 \mathrm{~mm}$ 


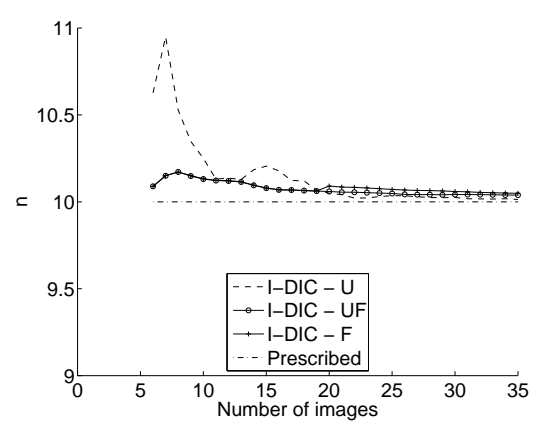

(a) $0.6 \%$ noise

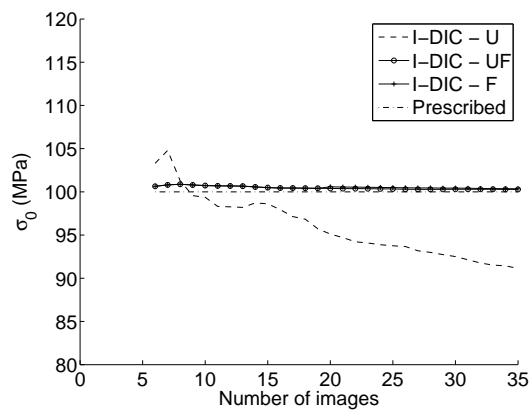

(c) $0.6 \%$ noise

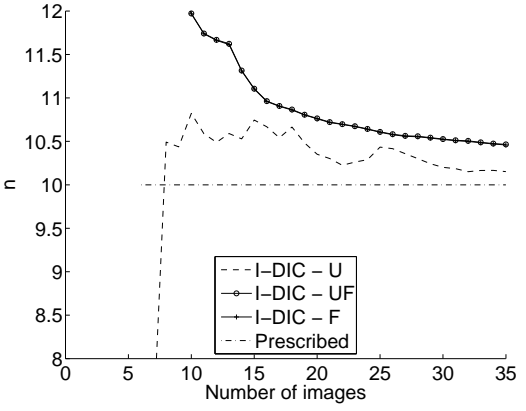

(b) $3 \%$ noise

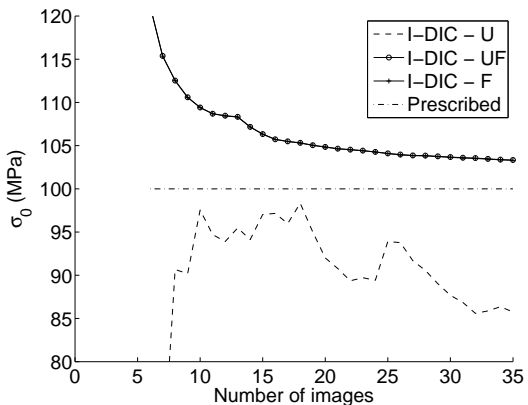

(d) $3 \%$ noise

Fig. 3 Identified parameters $n$ (top row (a) and (b)) and $\sigma_{0}$ in MPa (bottom row (c) and (d)). The left graphs ((a) and (c)) correspond to standard noise level $(0.6 \%$ of the dynamic range of $f$ ). The right graphs ((b) and (d)) show the corresponding identified values for a high noise level ( $3 \%$ of the dynamic range of $f$ ) 


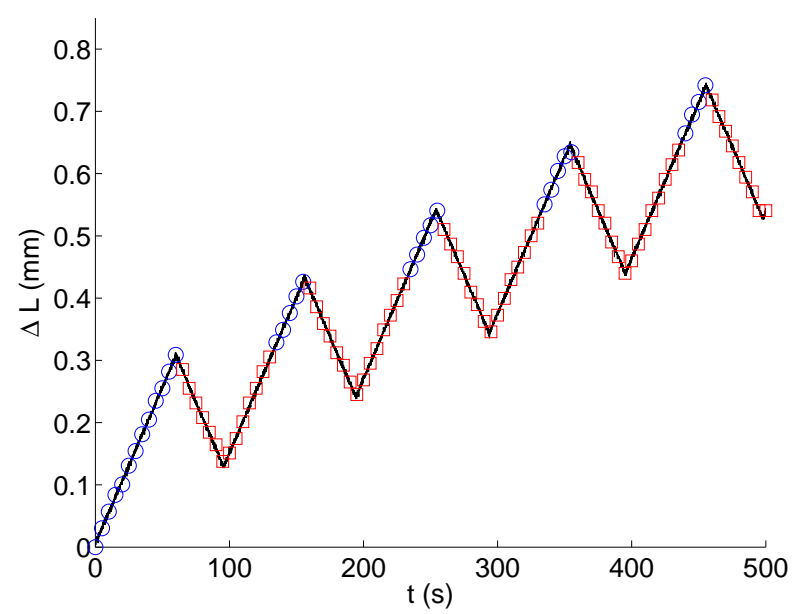

(a)

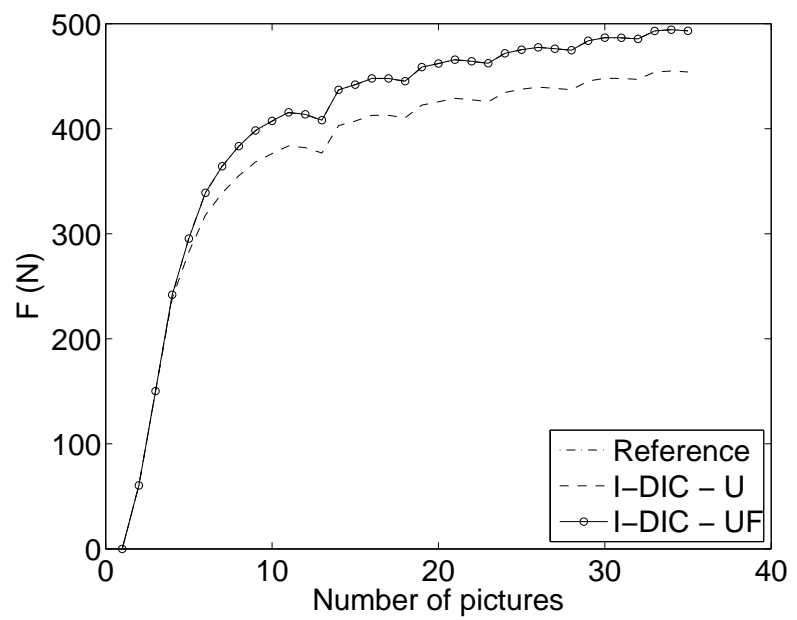

(b)

Fig. 4 (a) Elongation history of the experiment reported hereafter. The blue circles are used as input for the test case. (b) Computed sum of the reaction forces of the numerical specimen for the reference computation, for the I-DIC-U and I-DIC-UF procedures. The reaction forces are computed using the parameters identified with 35 images. The use of $\chi_{F}^{2}$ is necessary to match the reference reaction forces. The load fluctuations are due to the fact that actual displacements are prescribed on the two boundaries shown in Figure 2 


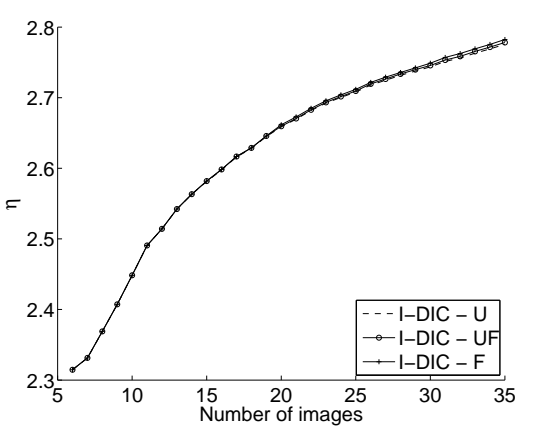

(a) $0.6 \%$ noise

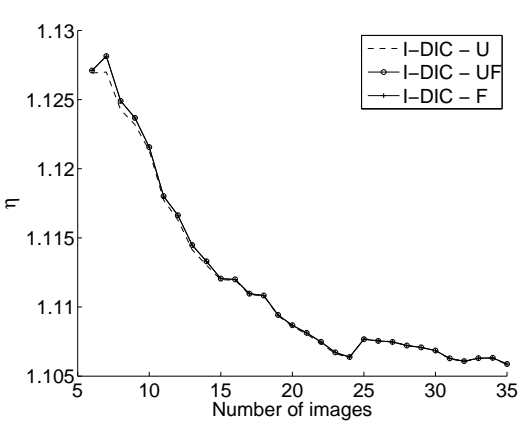

(b) $3 \%$ noise

Fig. 5 Correlation residuals $\eta$ obtained for the identified parameters using the three U, F and UF procedures. The left graph (a) corresponds to a standard noise level ( $0.6 \%$ of the dynamic range of $f$ ) and the right graph (b) to a large noise level (3\% of the dynamic range of $f$ )

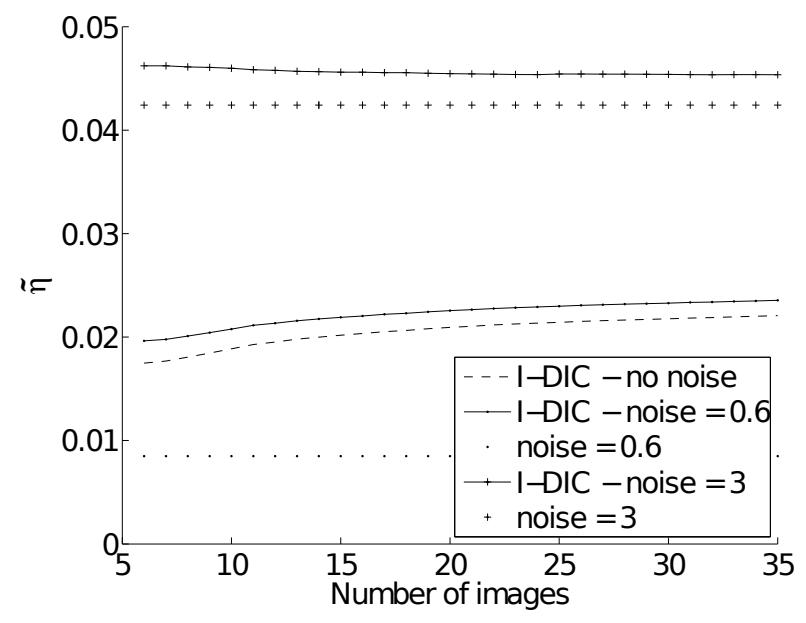

Fig. 6 Dimensionless correlation residual $\tilde{\eta}$ for the test-case for different noise levels. As the identified model is that used to deform the pictures, the error sources are image noise and gray level interpolations. For zero and low noise levels, the gray level interpolation error is predominant 


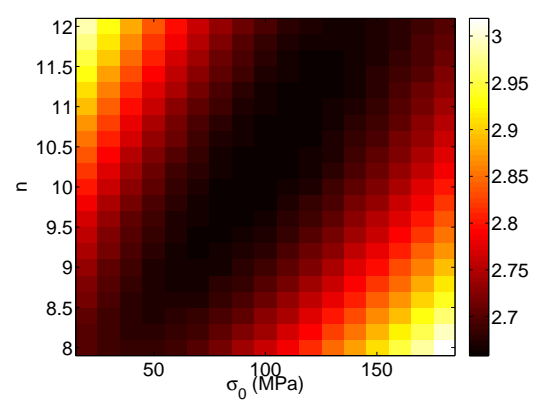

(a) $\eta$-map

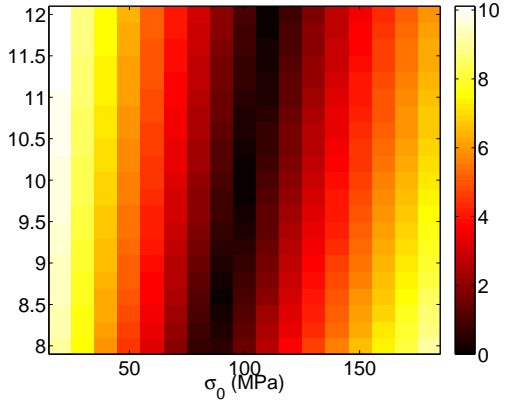

(b) $\chi_{F-\text { map }}$

Fig. 7 Correlation residual $\eta$ (a) and load residual $\chi_{F}$ (b) as functions of the value of the plastic parameters $\sigma_{0}$ and $n$ when the reference values are equal to $100 \mathrm{MPa}$ and 10 , respectively, and $0.6 \%$ noise is applied 


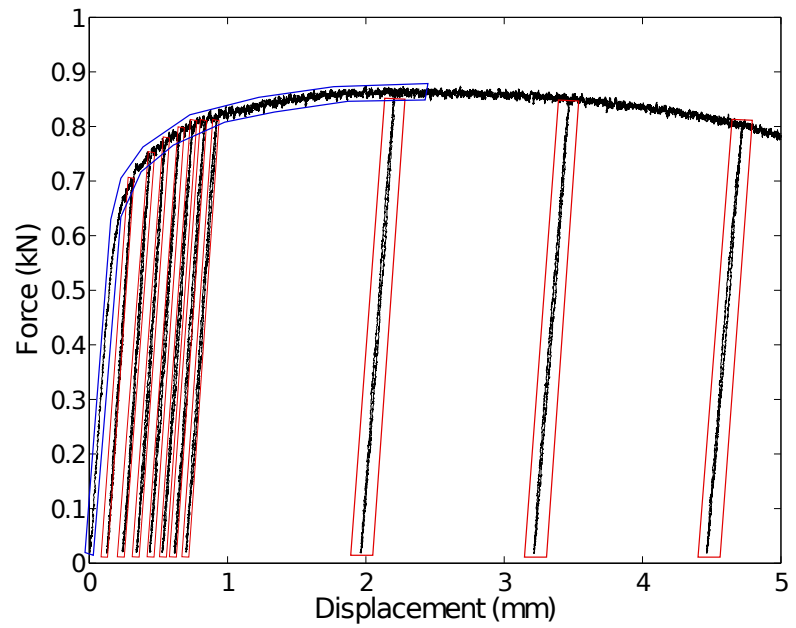

Fig. 8 Plot of the load/displacement curve. The red zones indicate the parts where images are selected for the determination of elastic parameters, the blue parts for plastic parameters 


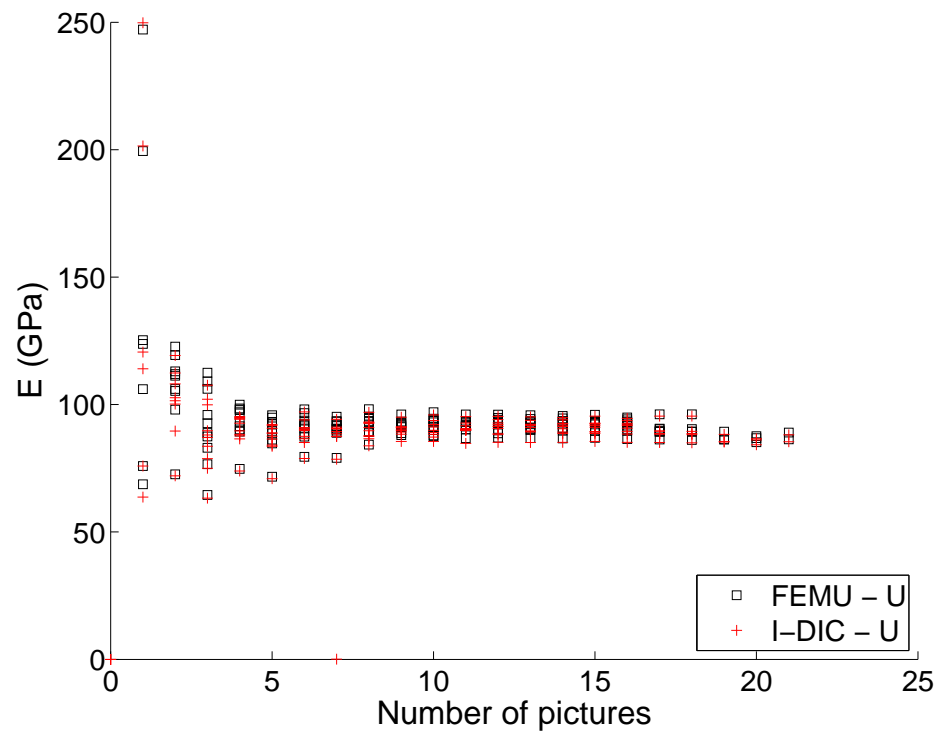

Fig. 9 Identified Young's modulus for 10 unloading/reloading sequences. Each point represents an evaluation of $E$ based on the two proposed identification methods and using different numbers of images in each sequence 


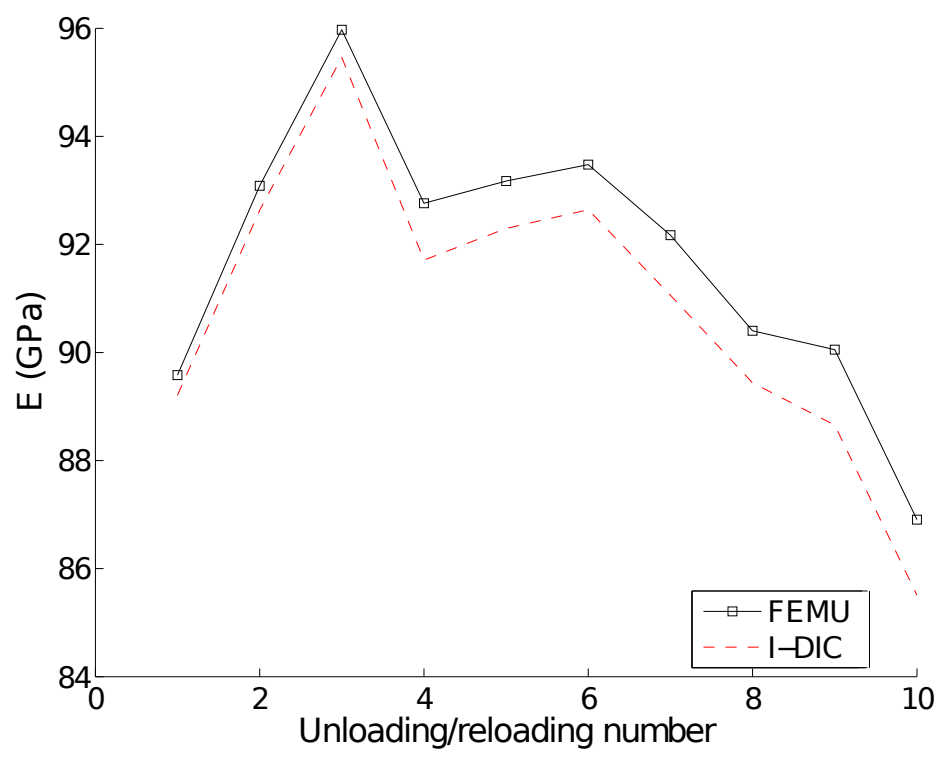

Fig. 10 Identified Young's moduli, for 15 images, for each of the 10 unloading/reloading sequences. A slight decrease may be observed and may be attributed to damage. However, because of the small amplitude of decrease, damage will not be considered 


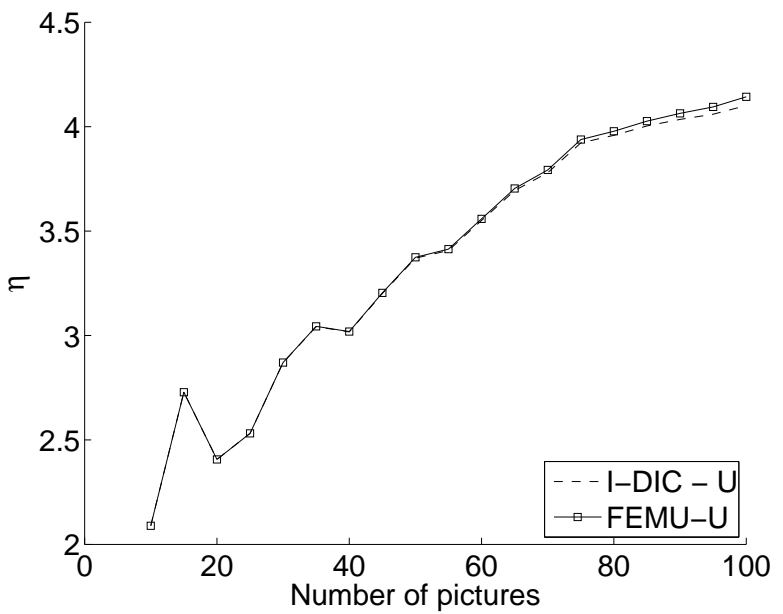

Fig. 11 Correlation residual $\eta$ after convergence of the algorithms. For FEMU-U, the level is an equivalent residual computed for one iteration of the I-DIC procedure after its convergence 

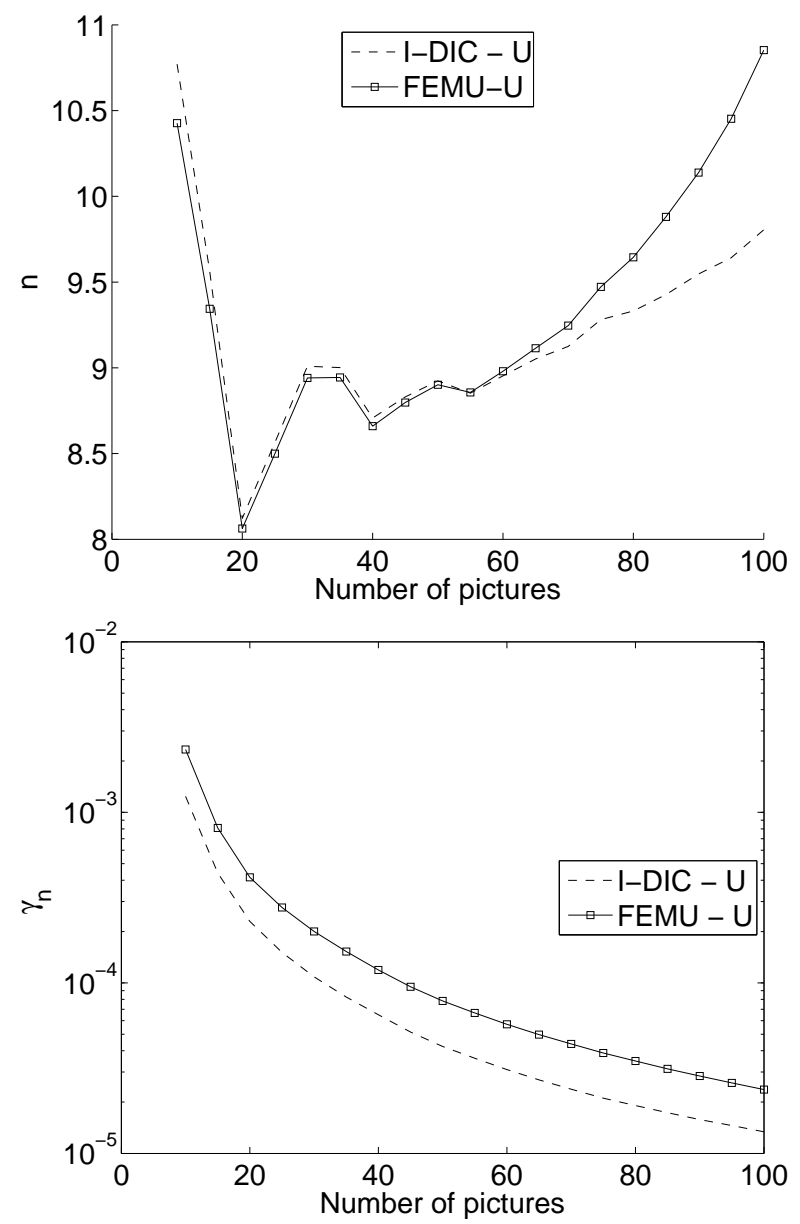

Fig. 12 Identification results for Ramberg-Osgood parameter $n$, and corresponding standard deviation due to noise. There are few differences between the results of both methods, except with a large number of images 

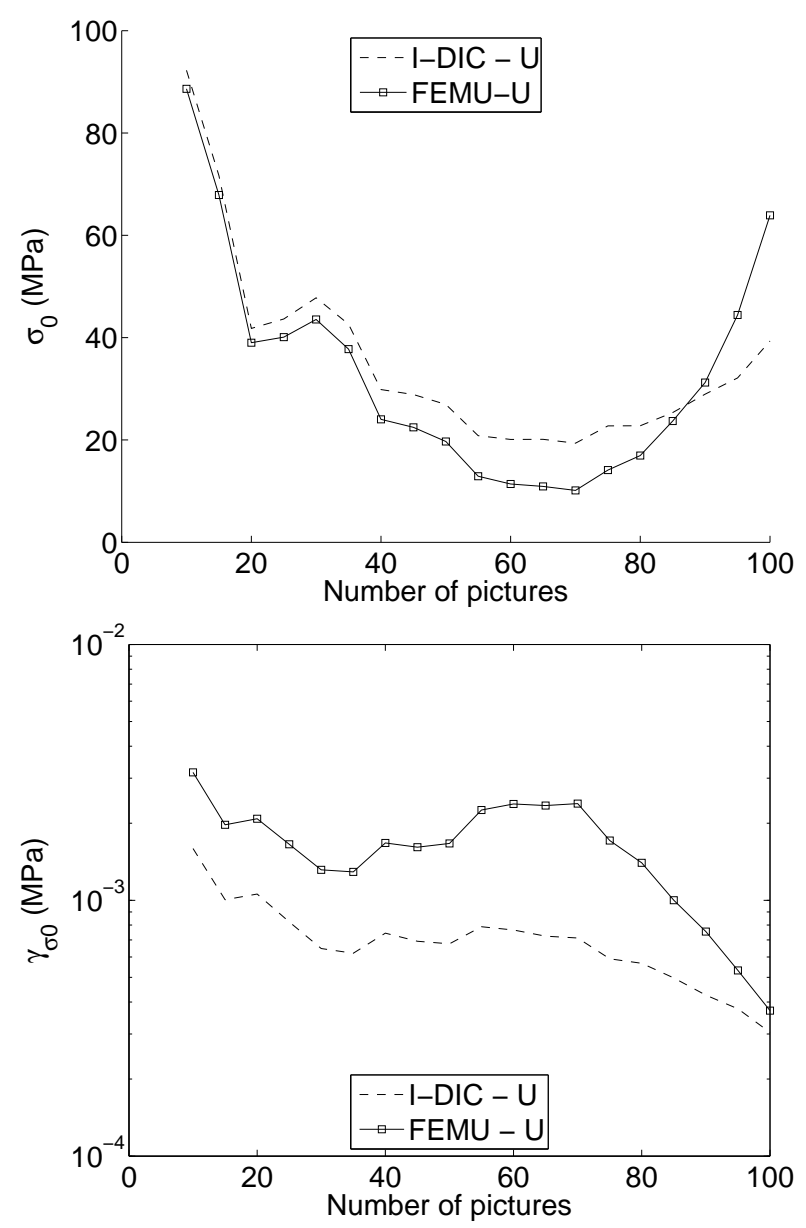

Fig. 13 Identification results for Ramberg-Osgood parameter $\sigma_{0}$, and corresponding resolution. The standard deviation due to noise being directly dependent on the parameter level, the inverse shape of the $\sigma_{0}$ curve can be observed in the standard deviation 


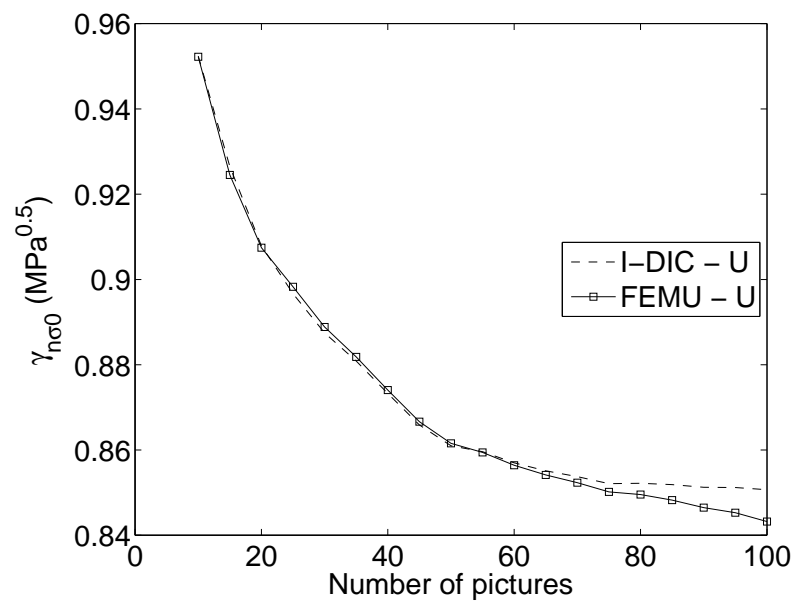

Fig. 14 Cross-correlation of the $\left(n, \sigma_{0}\right)$ pair. As in the test-case, its level is very high (around 0.9) and influences the identification of both parameters 


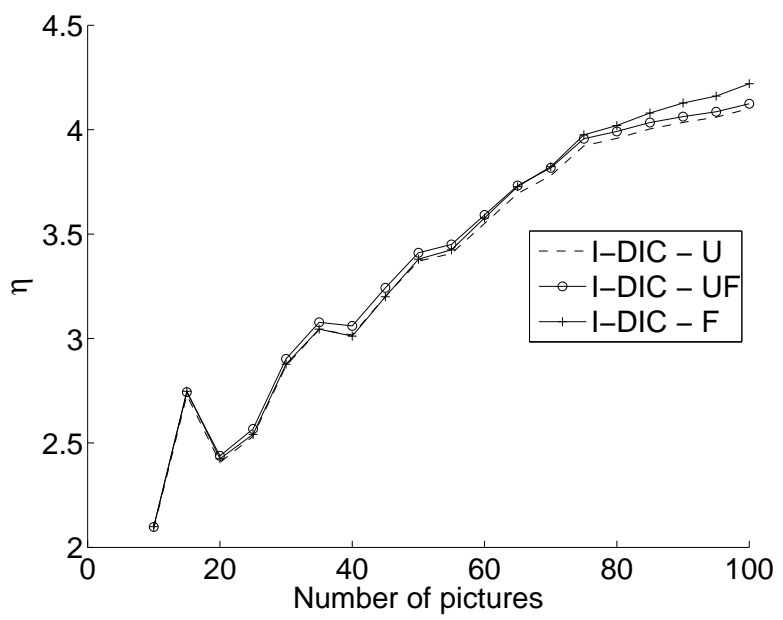

Fig. 15 Correlation residual $\eta$ after convergence of the algorithms. The residual is very similar with or without the use of load information

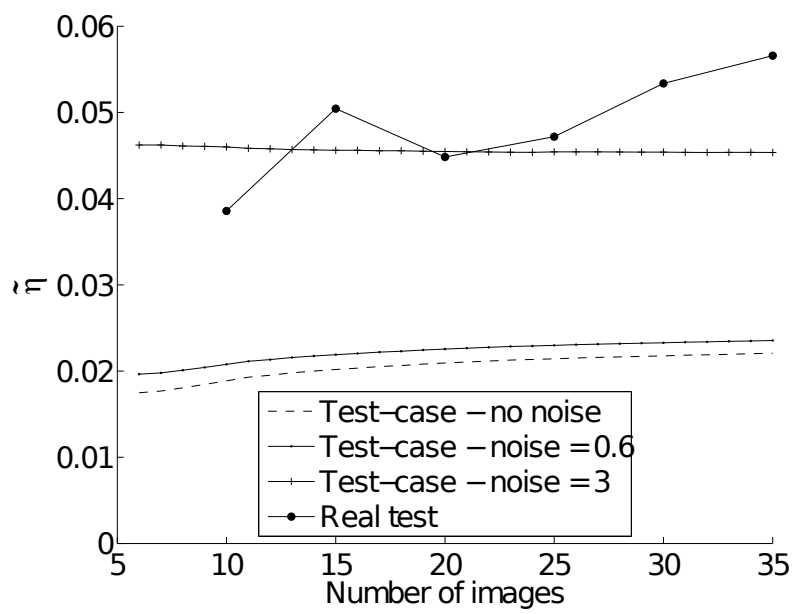

Fig. 16 Dimensionless correlation residuals $\tilde{\eta}$ for the test-case and the actual experiment. The results for the actual experiment show a significant drift that is not to be expected for this level of noise except if a model error arises 

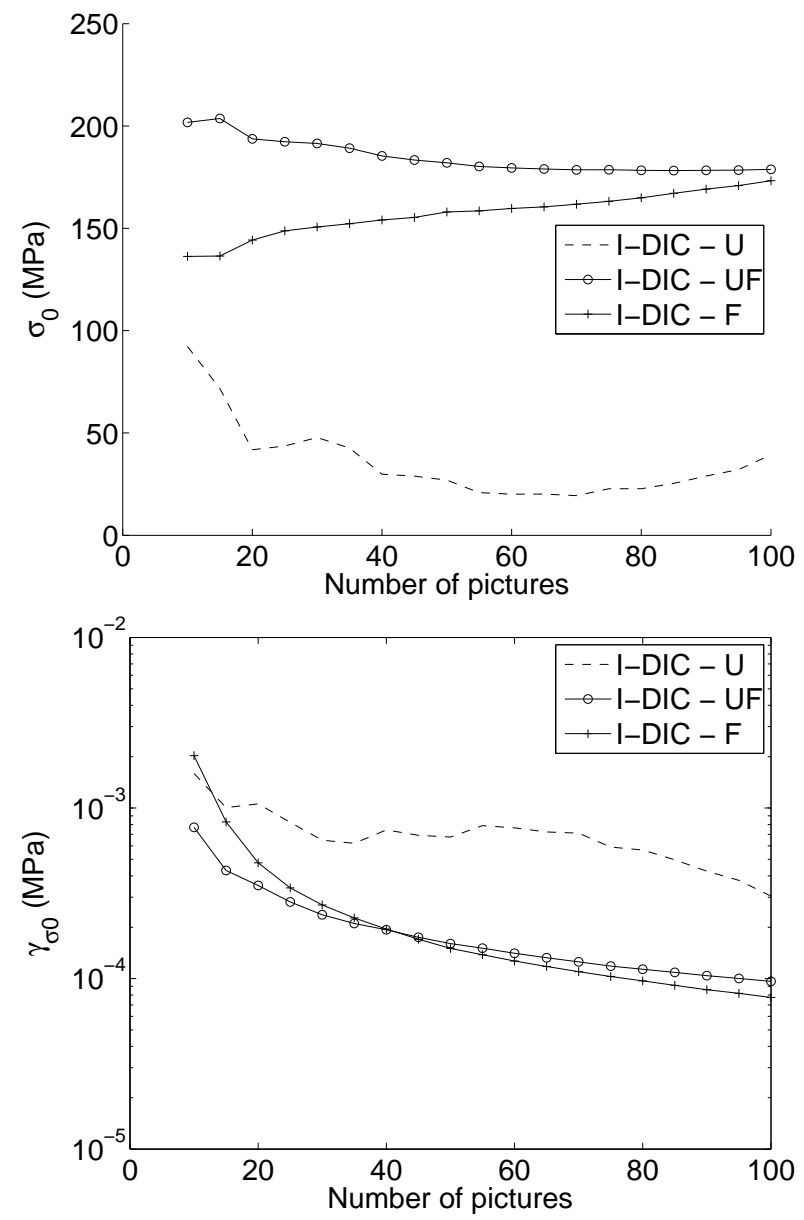

Fig. 17 Identification results for parameter $\sigma_{0}$, and corresponding resolution. The use of the equilibrium gap functional $\chi_{F}$ allows a steadier value of $\sigma_{0}$ to be identified and a lower standard resolution 

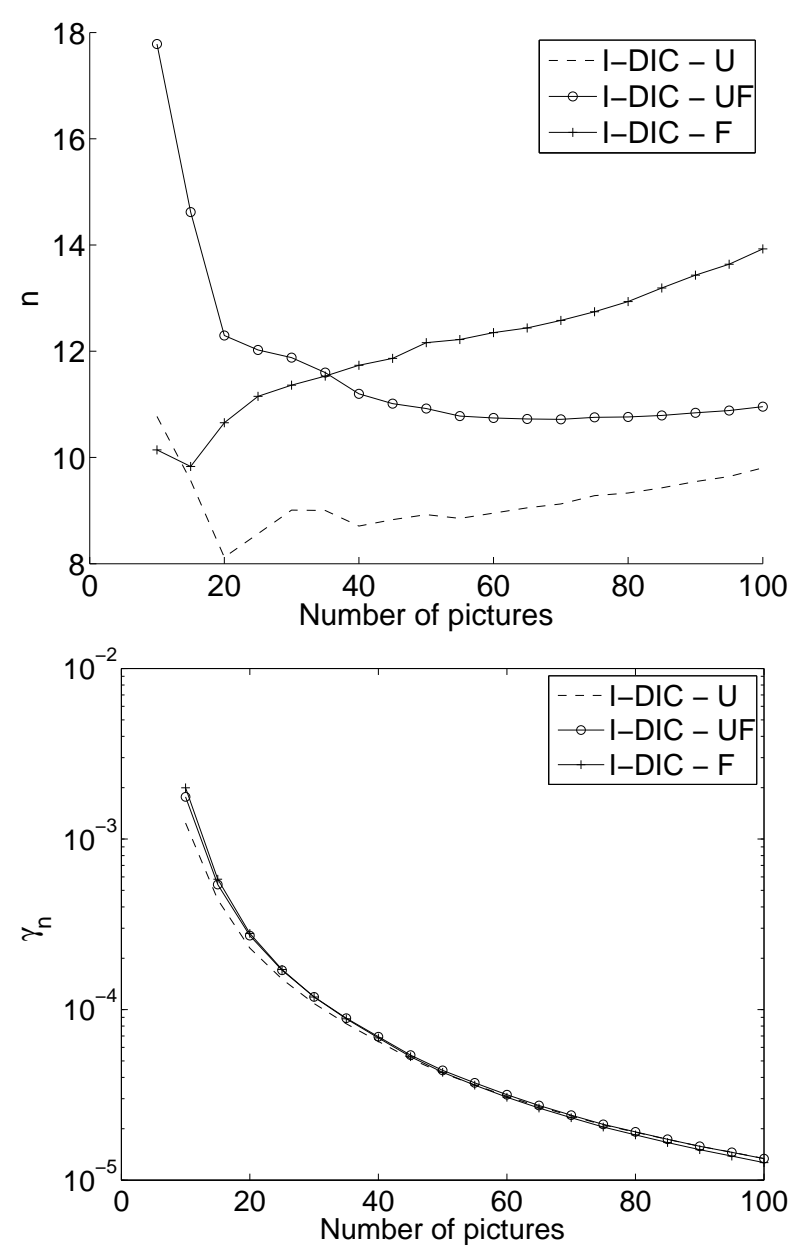

Fig. 18 Identification results for parameter $n$, and corresponding resolution. The use of the force function $\chi_{F}$ changes significantly the identified values of $n$ but not its standard resolution 


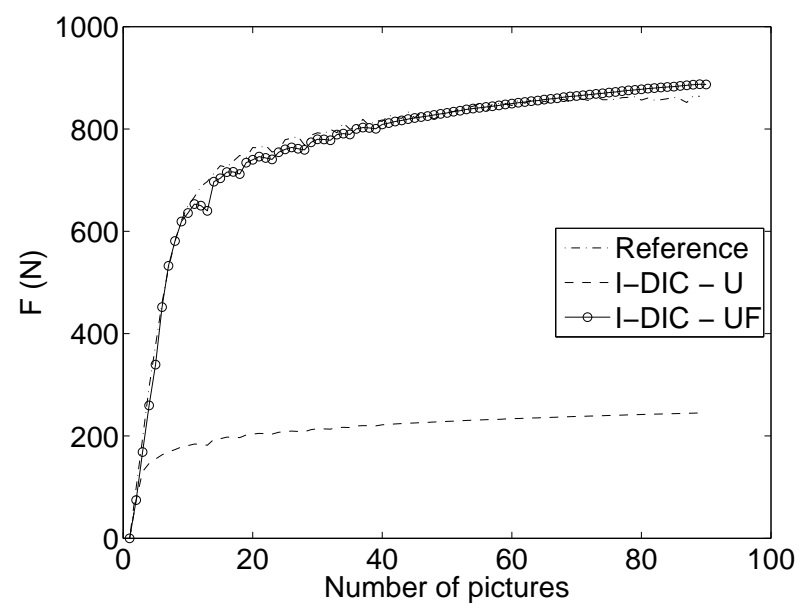

Fig. 19 Measured force and computed sum of the reaction forces for the I-DIC-U and I-DIC-UF procedures. The reaction forces are computed using the parameters identified with 90 images. This result shows that using only $\eta$ can lead to poor results in the present case 

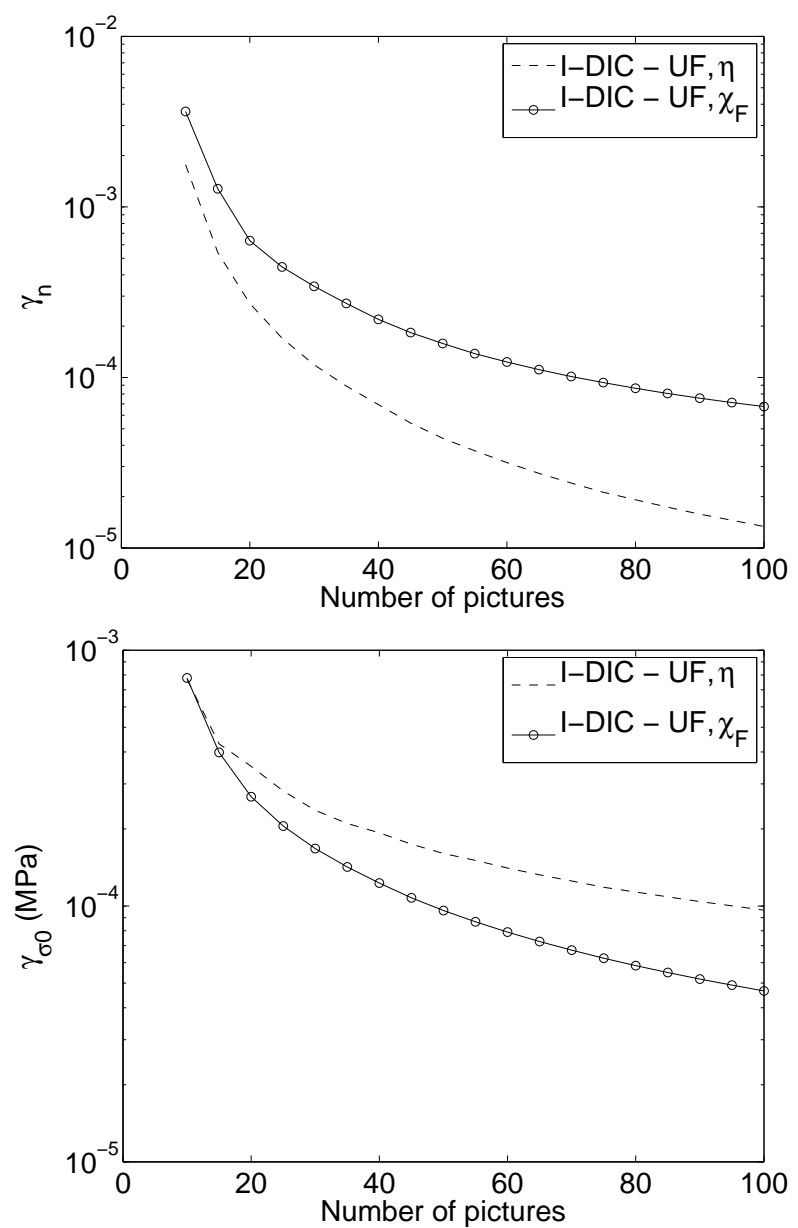

Fig. 20 Standard resolutions derived from the covariance matrices of $\eta$ and $\chi_{F}$ for parameters $n$ and $\sigma_{0}$ 